Original Article

\title{
DESIGN, SYNTHESIS, MOLECULAR DOCKING, ADMET STUDIES AND BIOLOGICAL EVALUATION OF PYRAZOLINE INCORPORATED 1, 2, 3-TRIAZOLE BENZENE SULPHONAMIDES
}

\author{
SRAVANTHI SILIVERI ${ }^{1,2^{*}}$, NAGARAJU BASHABOINA1, HARINADHA BABU VAMARAJU' ${ }^{1}$, SHIVA RAJ ${ }^{3}$ \\ 1Department of Pharmaceutical Chemistry, G. Pulla Reddy College of Pharmacy, Mehdipatnam, Hyderabad, Telangana, 500028, India, \\ ${ }^{2}$ Department of Pharmacy, OUCT, Osmania University, Hyderabad, 500007, India, ${ }^{3}$ Department of Chemistry, Osmania University, \\ Hyderabad, 500007, India \\ Email: sravanthisiliveri@gmail.com
}

Received: 19 Feb 2019 Revised and Accepted: 16 Apr 2019

\section{ABSTRACT}

Objective: The main objective of this work was to design, synthesize and evaluate the novel pyrazoline incorporated 1,2,3-triazole benzene sulphonamides for cytotoxic and anti-gout activities also to perform Insilco molecular docking studies.

Methods: Designed compounds were synthesized by condensation of different substituted chalcones (3a-i) with hydrazine hydrate and substituted phenylhydrazines. All the synthesized compounds were characterized on the basis of physical and spectral data. To predict the affinity and activity of the ligand molecule Libdock program was employed to generate different bioactive binding poses of designing molecules at the active site of protein Phosphatidylinositol 3-kinase (PI3K $\alpha$ ). Title compounds were evaluated for cytotoxic activity by using 3-(4, 5-dimethylthiazol-2-yl)-2,5diphenyltetrazolium bromide (MTT) assay and anti-gout activity by potassium oxonate induced assay.

Results: All the synthesized compounds showed characteristic peaks in FTIR, ${ }^{1} \mathrm{H},{ }^{13} \mathrm{C}$ NMR and MASS spectral analysis. In molecular docking studies, compound $3 \mathrm{i}$ has shown good binding affinity to the active site of PI3Ka with a docking score of 145.031 and 4 hydrogen bonding interactions with least hepatotoxicity and good bioavailability when compared with that of reference ligand KKR exhibited a Libdock score of 88.35. Remaining compounds also have a good binding affinity with a minimum of 2 bonding interactions and having better absorption, distribution, metabolism, elimination and toxicity (ADMET) profile. The same compound (3i) exhibited the highest cytotoxic activity with an $\mathrm{IC}_{50} \mathrm{value}$ of $4.54 \mu \mathrm{g} / \mathrm{ml}$. Compound $4 \mathrm{~d}$ was evaluated for anti-inflammatory activity and it has significantly ameliorated against potassium oxonate induced gout in mice when compared with that of standard drug allopurinol due to its anti-inflammatory property.

Conclusion: We designed and synthesized a novel series of title compounds in quantitative yields and performed docking studies. New derivatives have a good binding affinity towards PI3K $\alpha$ enzyme, good bioavailability, least hepatotoxicity and significant cytotoxic activity.

Keywords: ADMET, Cancer, Docking, Malignant, PI3K $\alpha$, Proliferation

(C) 2019 The Authors. Published by Innovare Academic Sciences Pvt Ltd. This is an open-access article under the CC BY license (http://creativecommons.org/licenses/by/4.0/) DOI: http://dx.doi.org/10.22159/ijpps.2019v11i6.32684

\section{INTRODUCTION}

Cancer is a multifactorial disease, arising from the uncontrolled proliferation of a cell with the potential to invade to other organs of the body [1]. PI3K $\alpha$ is one of the proto-oncogenes, which has a vital role in the regulation of many important cell signaling pathways including cellular replication, cell proliferation leading to growth and apoptosis $[2,3]$. The enzyme PI3K $\alpha$ appeared to involve in $40 \%$ of all types of human cancers including breast, gastric, cervical, urinary tract, colon, non-small-cell lung and squamous cell lung carcinomas [4]. Considerable evidence suggest that PI3K (P110 $\alpha)$ subunit protein is expected to mutate in these cancers that bring about constitutive downstream pathways leading to defective control of cellular proliferation and malignant transformation, thus $\mathrm{PI} 3 \mathrm{~K} \alpha$ is drawing attention in cancer biology $[5,6]$. Hence targeting $\mathrm{PI} 3 \mathrm{~K} \alpha$ may be considered as a promising approach in the design of new anticancer drugs.

Pyrazoles are a class of heterocyclic compounds characterized by 5membered aromatic ring structure composed of three carbon atoms and two nitrogen atoms in adjacent positions. Pyrazole derivatives have a long history of applications in agriculture as herbicides and insecticides as well as in the pharmaceutical industry as antipyretic and anti-inflammatory agents [7-12]. Pyrazole derivatives have been reported to show a broad spectrum of biological activity including antibacterial [13], antifungal [14], analgesic [15], anti-inflammatory [16-18], neuroprotective [19], estrogen receptor binding [20], antineoplastic [21], activities. Due to their wide range of biological activities, pyrazoles received considerable interest in the field of drug discovery and therefore pyrazole ring constitutes a relevant synthetic target in the pharmaceutical industry. On the other hand, 1,2,3-triazole is an important heterocycle which has gained a lot of interest for researchers in view of its high potency, low toxicity with broad spectrum of activities. Triazole derivatives have been reported to have anticancer [22], anti-inflammatory [23], antibacterial [24], antiviral [25], anti-human immuno virus (HIV) [26], fungicidal [27] and insecticidal [28-30] activities. The objective of the current work was to synthesize novel pyrazoline incorporating 1,2,3-triazole benzene sulphonamide derivatives as potent antiproliferative and anti-inflammatory agents. We were well known about the synthesis of simple pyrazolines from chalcones but we designed pyrazoline incorporating 1,2,3-triazolyl benzene sulphonamide derivatives. Inspired by the biological properties of both pyrazole and triazole heterocycles in the present study, we thought of synchronizing both these moieties into a single molecule in order to obtain new hybrid molecules with improved biological activity and low toxicity.

\section{MATERIALS AND METHODS}

All the chemicals and solvents used were of synthetic grade from SD fine chemicals Ltd., (Mumbai, India), and avra chemicals pvt ltd Hyderabad. Completion of the reaction was monitored by analytical thin layer chromatography (TLC) using E-Merck $0.25 \mathrm{~mm}$ silica gel plates. Visualization was accomplished with ultraviolet (UV) light (256 $\mathrm{nm})$ and iodine chamber. Synthesized compounds were purified by the re-crystallization process. The purity of the compounds was checked by a single spot in TLC and solvent system 
for TLC was determined on trial and error basis. Melting points were determined in open capillary tubes using ANALAB melting point apparatus and were uncorrected. All the ${ }^{1} \mathrm{H}$ NMR spectra were recorded on varian $400 \mathrm{MHz}$ spectrometer using DMSO- $\mathrm{d}_{6}$ as solvent and tetramethylsilane (TMS) as an internal standard. Chemical shift values are listed in $\delta$ scale. The FT-IR spectra were recorded on schimadzu FT-IR spectrophotometer by using $1 \%$ potassium bromide discs. Mass spectra of the compounds were recorded on electronic spin ionization mass spectra (ESI-MS) on aglient 1100 series.

\section{Synthesis of 4-(4-acetyl-5-methyl-1H-1,2,3-triazol-1-yl) benzene} sulfonamide (2)

Sodium metal was dissolved in $10 \mathrm{ml}$ of absolute ethanol in a dry flask to which azido benzene sulfonamide and $2 \mathrm{ml}$ of acetylacetone were added and stirred for $1 \mathrm{~h}$ on magnetic stirrer. Transferred to the round-bottomed flask (RBF) and refluxed at $140{ }^{\circ} \mathrm{C}$ for $12-16 \mathrm{~h}$. The completion of the reaction was monitored by TLC. The reaction mixture was poured into crushed ice to obtain the solid product. Then the precipitate was filtered under suction, washed thoroughly with water and recrystallized from aq. methanol.

Synthesis of substituted 4-(4-(3-(phenyl)acryloyl)-5-methyl1H-1,2,3-triazol-1-yl)benzenesulfonamide derivatives 3(a-i)

Different substituted benzaldehydes $(0.01 \mathrm{~mol})$ and 4-(4-acetyl-5methyl-1H-1,2,3-triazol-1-yl)benzenesulfonamide 2 ( $0.01 \mathrm{~mol})$ were dissolved in $95 \%$ of ethanol in a conical flask to this added $\mathrm{NaOH}$ pellet the reaction mixture was stirred for $30 \mathrm{~min}$. The solid obtained was collected and recrystallized from absolute ethanol.

Synthesis of substituted 4-(4-(5-(phenyl)-4,5-dihydro-1H-pyrazol3-yl)-5-methyl-1H-1,2,3-triazol-1-yl)benzenesulfonamide derivatives $4(a-1)$

Substituted chalcones $3(\mathrm{a}-\mathrm{o})$ ( $0.01 \mathrm{~mol})$, hydrazine hydrate $(0.015$ $\mathrm{mol}$ ) were mixed in ethanol in a RBF and refluxed for $3 \mathrm{~h}$. The completion of the reaction was monitored by TLC. The reaction mixture was poured into crushed ice to obtain the solid product. Solid was collected and recrystallized from methanol.

Synthesis of substituted 4-(5-methyl-4-(5-phenyl-4,5-dihydro1H-pyrazol-3-yl)-1H-1,2,3-triazol-1-yl)benzenesulfonamide 5(a-i)

Substituted chalcones 3(a-o) (0.01 mol), substituted phenyl hydrazines $(0.01 \mathrm{~mol})$ and a catalytic amount of glacial acetic acid were refluxed in ethanol in RBF for 4-5 h After completion of the reaction, the reaction mixture was poured into crushed ice to obtain the solid product. Then solid was collected and recrystallized from aq. ethanol.

\section{Molecular docking and ADMET studies}

The molecular docking study of synthesized compounds was done by employing the Libdock protocol in order to identify binding interactions with the target protein PI3K $\alpha$ (PDB ID: 3ZIM). ADMET studies were carried out by using discovery studio software.

\section{Cytotoxic activity}

The title compounds were evaluated for In vitro antiproliferative activity via MTT ([3-(4, 5-dimethylthiazol-2-yl)-2,5-diphenyl tetrazolium bromide]) based cytotoxic assay[31] against MCF-7 breast cancer cell line with taxol as standard reference. Cell lines were purchased from the national institute of nutrition (NIN) Hyderabad. Cells were harvested from the logarithmic phase of cultures and re-suspended in Dulbecco's Modified Eagle's Medium supplemented with $10 \%$ fetal bovine serum (FBS). The cell counts were adjusted and equal number of cells were plated into each well of 96-well culture plates and allowed to grow overnight at $37^{\circ} \mathrm{C}$, in presence of $5 \% \mathrm{CO}_{2}$. The cells were treated with test substances at various concentrations as indicated for $72 \mathrm{~h}$. In vehicle control culture wells, a maximum of $0.5 \%$ DMSO was added. Culture medium was renewed at every $24 \mathrm{~h}$ with fresh culture medium supplemented with test substances. Thereafter, $0.5 \mathrm{mg} / \mathrm{ml}$ of MTT reagent was added to each well and the microplate was incubated further for $4 \mathrm{~h}$ at $37{ }^{\circ} \mathrm{C}$ in presence of $5 \% \mathrm{CO}_{2}$. Finally, the cells were solubilized by adding solubilizing solution and allowed to incubate at $37{ }^{\circ} \mathrm{C}$ overnight. After complete solubilization of the formazan crystals the absorbance was read at $540 \mathrm{~nm}$ in a microplate reader (Bio-Rad, USA). The results (mean $\mathrm{OD} \pm \mathrm{SD}$ ) obtained from quadruplicate wells were used in the calculation to determine the cytotoxicity $\left(50 \%\right.$ of inhibitory concentration, $\left.\mathrm{IC}_{50}\right)$ of the test compounds.

\section{Evaluation of anti-inflammatory effect of compound $4 \mathrm{~d}$ against potassium oxonate induced gout in mice}

Anti-inflammatory activity of compound $4 \mathrm{~d}$ in potassium oxonate induced gout in mice was investigated in the present study. Intraperitoneal injections of potassium oxonate $250 \mathrm{mg} / \mathrm{kg}$ daily for $28 \mathrm{~d}$ has lead to increased levels of serum concentration of creatinine, uric acid, blood urea nitrogen, and xanthine oxidase enzyme level as an indicator of impaired kidney function and increased plasma concentration of the blood parameters such as total leukocyte count, differential leukocyte count and erythrocyte sedimentation rate (ESR) indicates inflammation. Treatment with compound $4 \mathrm{~d}$ in doses $50 \mathrm{mg} / \mathrm{kg}$ and $100 \mathrm{mg} / \mathrm{kg}$ for $28 \mathrm{~d}$ exhibited a significant improvement in gout disease in mice as evidenced by the decrease in biochemical parameters and inflammation in joints. The total leukocyte count, differential leukocyte count and erythrocyte sedimentation rate were estimated as per reported methods. Creatinine level was determined in serum by using modified jaffe's reaction. The level of xanthine oxidase was determined by. Uric acid level was determined in serum by using peroxidase endpoint assay. Blood urea nitrogen level was determined by using berthelot end point assay. Present study indicated that the group of mice given standard reference compound allopurinol at $5 \mathrm{mg} / \mathrm{kg}$ body weight significantly $(\mathrm{p}<0.05)$ reduced serum creatinine, uric acid, blood urea nitrogen, and xanthine oxidase enzyme level in hyperuricemic mice.

Male albino mice were obtained from Sainath Agencies Hyderabad. They were acclimatized to laboratory conditions $\left(22 \pm 2{ }^{\circ} \mathrm{C}\right.$ of temperature, 12-hr light/dark cycle), food and water were given ad libitum. After an acclimatization period of $2 \mathrm{w}$, they were randomly divided into 5 experimental groups.

To evaluate Anti-inflammatory effect of Quinoline-3-carbonitrile derivative against potassium oxonate induced gout in mice. 30 Male albino mice weighing 25-30 gm were randomly divided into five groups of six mice in each group such as:

Group 1: Normal control (received saline p. o daily for $28 \mathrm{~d}$ ).

Group 2: Disease control (received potassium oxonate $250 \mathrm{mg} / \mathrm{kg}$ i. $\mathrm{p}$ for $28 \mathrm{~d}$ ).

Group 3: Standard control (received potassium oxonate $250 \mathrm{mg} / \mathrm{kg}$ i. p and allopurinol $5 \mathrm{mg} / \mathrm{kg} \mathrm{p}$. o for $28 \mathrm{~d}$ ).

Group 4: Low dose group (received potassium oxonate $250 \mathrm{mg} / \mathrm{kg}$ i. $\mathrm{p}$ and Quinoline-3-carbonitrile derivative $50 \mathrm{mg} / \mathrm{kg} \mathrm{p}$. o for $28 \mathrm{~d}$ ).

Group 5: High dose group (received potassium oxonate $250 \mathrm{mg} / \mathrm{kg}$ i. $p$ and Quinoline-3-carbonitrile derivative $100 \mathrm{mg} / \mathrm{kg} \mathrm{p}$. o for $28 \mathrm{~d}$ ).

All the groups except normal control have received potassium oxonate $250 \mathrm{mg} / \mathrm{kg}$ intraperitoneally for $28 \mathrm{~d}$ and 1 hour later, standard group and treatment groups have received allopurinol (5 $\mathrm{mg} / \mathrm{kg} \mathrm{p.} \mathrm{o)} \mathrm{and} \mathrm{quinoline-3-carbonitrile} \mathrm{(low} \mathrm{dose-} 50 \mathrm{mg} / \mathrm{kg}$ and high dose-100 mg/kg p. o) respectively. Differential leukocyte count and total leukocyte count were estimated on $7^{\text {th }}, 14^{\text {th }}$ and $29^{\text {th }}$ day by collecting blood through retro-orbital plexus. On $29^{\text {th }}$ day blood samples were collected for estimating biochemical parameters and later on all animals were sacrificed for histopathological studies.

All the experimental procedures were carried out in accordance with the committee for the purpose of control and supervision of experiments on animals (320/CPCSCEA dated 03-01-2001) guidelines. The study was reviewed and approved by the Institutional Animal Ethics Committee (GPRCP/IAEC) 10/18/ 02/PCL/AE-2-Mice-M/F-30), G. Pulla Reddy College of Pharmacy, Mehdipatnam, Hyderabad, India. 


\section{RESULTS AND DISCUSSION}

\section{Chemistry}

The synthesis of compounds $4(\mathrm{a}-\mathrm{l})$ and $5(\mathrm{a}-\mathrm{i})$ is given under fig 1 . In this scheme, the intermediate 4-(4-acetyl-5-methyl-1H-1,2,3-triazol-1yl)benzenesulfonamide (2) was synthesized by diazotization of 4amino benzene sulphonamide and followed by condensation of 4azidobenzene sulphonamide with acetylacetone in presence of sodium ethoxide under reflux for 12-15 h. Claisen Schmidt condensation reaction of intermediate (2) with various substituted aromatic aldehydes under basic conditions gave chalcones 3(a-l). In the final step, reaction of chalcones $3(\mathrm{a}-\mathrm{l})$ with hydrazine hydrate in ethanol under reflux for 3-4 h gave 4-(5-methyl-4-(5-phenyl-4,5-dihydro- $1 \mathrm{H}$ -
pyrazol-3-yl)-1H-1,2,3-triazol-1-yl) benzene sulfonamides 4(a-l) (Step $4 \mathrm{~A}$ ) in good yields. Simultaneously, intermediate (3) condensed with different substituted phenylhydrazines in ethanol under reflux for 4 $5 \mathrm{~h}$ to obtain 4-(5-methyl-4-(5-phenyl-4,5-dihydro-1H-pyrazol-3yl)-1H-1,2,3-triazol-1-yl)benzenesulfonamides of series-2 [5(a-i)] (Step 4B) in good yields. All the derivatives were characterized by ${ }^{1} \mathrm{H}$ NMR, IR and ESI-MASS spectral data.

The ${ }^{1} \mathrm{H}$ NMR spectrum of final compounds contains a singlet around $2.61 \mathrm{ppm}$ for $\mathrm{CH}_{3}$ protons, protons of $\mathrm{CH}_{2}$ and $\mathrm{CH}$ of pyrazoline ring appeared as doublet of doublets, triplet of doublets in the range of 3 $5 \mathrm{ppm}$, aromatic protons appeared in the range of 6.5-8.2 ppm and two protons of $-\mathrm{NH}_{2}$ appeared as a singlet at $7.6 \mathrm{ppm}$ whereas one proton of pyrazoline $\mathrm{NH}$ appeared as singlet at $7.7 \mathrm{ppm}$.

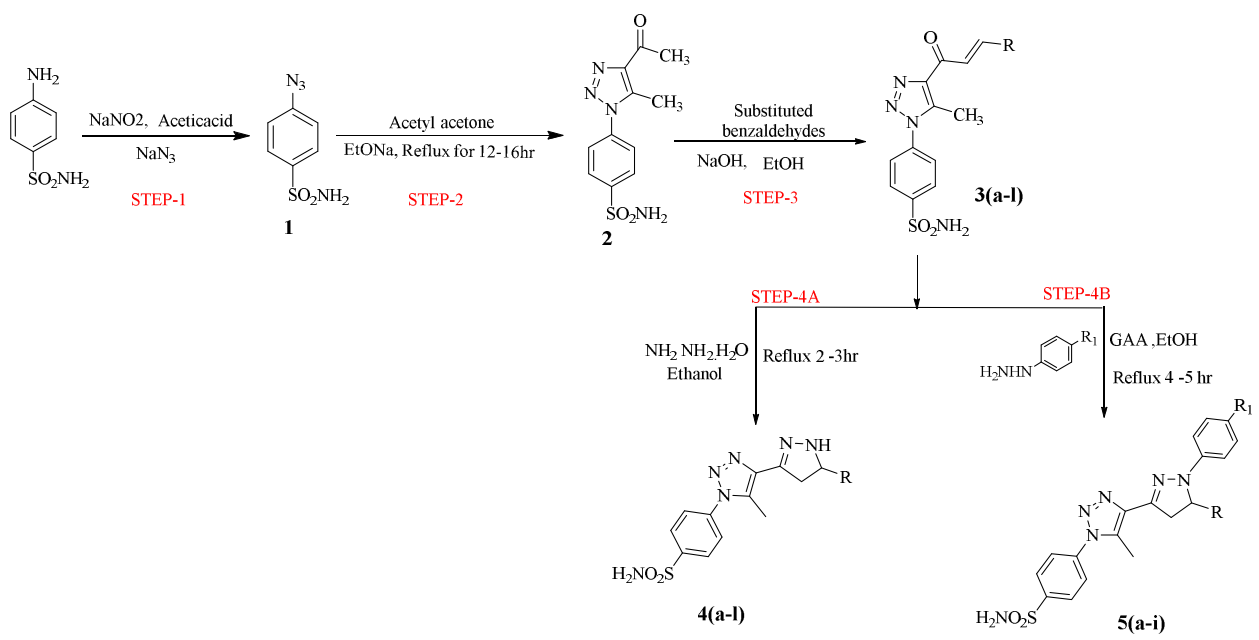

Where $\mathbf{R}$ and $\mathbf{R}_{1}=$

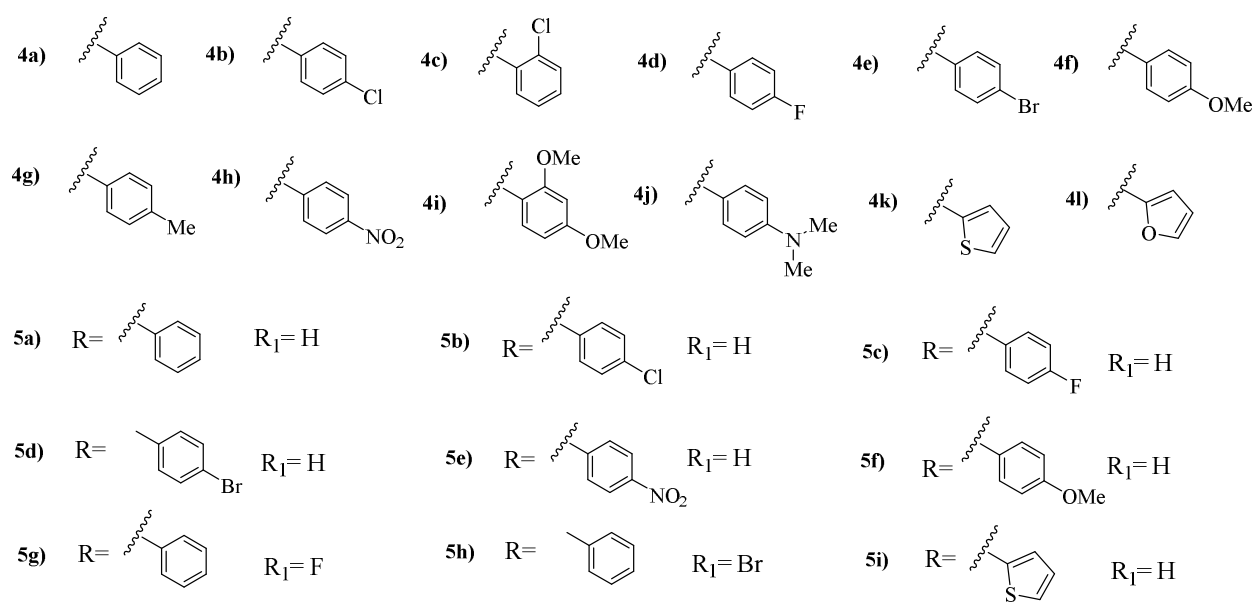

Fig. 1: Synthesis of pyrazoline incorporated 1,2,3-triazole benzene sulphonamide derivatives

\section{Spectral characterization of synthesized compounds}

4-(4-Acetyl-5-methyl-1H-1,2,3-triazol-1-yl)benzenesulfonamide (2): Yield 95\%, mp $129-131^{\circ} \mathrm{C}$. IR spectrum, $v \mathrm{~cm}^{-1}: 1174,1357$, 1689, 3192, 3273. ${ }^{1} \mathrm{H}$ NMR ( $\left.400 \mathrm{MHz}, \mathrm{DMSO}-\mathrm{d}_{6}\right): 2.56\left(\mathrm{~s}, 3 \mathrm{H}, \mathrm{CH}_{3}\right)$, $2.65\left(\mathrm{~s}, 3 \mathrm{H}, \mathrm{COCH}_{3}\right), 7.61\left(\mathrm{~s}, 2 \mathrm{H},-\mathrm{NH}_{2}\right), 7.88(\mathrm{dd}, 2 \mathrm{H}, \mathrm{ArH}, J=6.9 \mathrm{~Hz})$, 8.08 (dd, $2 \mathrm{H}, \mathrm{ArH}, J=9.0 \mathrm{~Hz}$ ). ESI-MS: m/z $281(\mathrm{M}+1)$ observed for $\mathrm{C}_{11} \mathrm{H}_{12} \mathrm{~N}_{4} \mathrm{O}_{3} \mathrm{~S}$.

4-(4-Cinnamoyl-5-methyl-1H-1,2,3-triazol-1-yl)benzene sulfonamide (3a): Yield 95\%, mp 340-342 ${ }^{\circ} \mathrm{C}$. IR spectrum, $v, \mathrm{~cm}^{-1}$ : 1174, 1357, 1656, 3192, 3273. ${ }^{1} \mathrm{H}$ NMR (400 MHz, DMSO-d $)$ ): 2.61 (s, $\left.3 \mathrm{H},-\mathrm{CH}_{3}\right), 7.51(\mathrm{t}, 3 \mathrm{H}, \mathrm{ArH}), 7.6\left(\mathrm{~s}, 2 \mathrm{H},-\mathrm{NH}_{2}\right), 7.75(\mathrm{~d}, 2 \mathrm{H}$, olefinic, $\mathrm{ArH})$, 7.8-8.05 (m, 6H, ArH and $\left.\mathrm{NH}_{2}\right)$. ESI-MS: $\mathrm{m} / \mathrm{z} 369(\mathrm{M}+1)$ observed for $\mathrm{C}_{18} \mathrm{H}_{16} \mathrm{~N}_{4} \mathrm{O}_{3} \mathrm{~S}$.
4-(4-(3-(4-Chlorophenyl)acryloyl)-5-methyl-1H-1,2,3triazol-1-yl)benzene sulfonamide (3b): Yield 97\%, mp 360$361^{\circ} \mathrm{C}$. IR spectrum, $v, \mathrm{~cm}^{-1}: 1091,1159,1315,1664,3304$, 3350. ${ }^{1} \mathrm{H}$ NMR (400 MHz, DMSO-d $): 2.61\left(\mathrm{~s}, 3 \mathrm{H}, \mathrm{CH}_{3}\right), 7.5(\mathrm{~d}, 2 \mathrm{H}$, $\mathrm{ArH}, J=6.9 \mathrm{~Hz}), 7.6(\mathrm{~d}, 2 \mathrm{H}, \mathrm{ArH}), 7.8-7.95\left(\mathrm{~m}, 7 \mathrm{H}, \mathrm{ArH}\right.$ and $\mathrm{NH}_{2}$, olefinic), $8.02(\mathrm{~d}, 1 \mathrm{H}$, olefinic). ESI-MS: $\mathrm{m} / \mathrm{z} 403(\mathrm{M}+1)$ observed for $\mathrm{C}_{18} \mathrm{H}_{15} \mathrm{ClN}_{4} \mathrm{O}_{3} \mathrm{~S}$.

4-(4-(3-(4-Methoxyphenyl)acryloyl)-5-methyl-1H-1,2,3triazol-1-yl)benzene sulfonamide (3c): Yield 98\%, mp 260$262{ }^{\circ} \mathrm{C}$. IR spectrum, $v, \mathrm{~cm}^{-1}: 1031,1164,1345,1654,3213$, 3396. ${ }^{1} \mathrm{H}$ NMR $\left(400 \mathrm{MHz}, \mathrm{DMSO}-\mathrm{d}_{6}\right): 2.61\left(\mathrm{~s}, 3 \mathrm{H}, \mathrm{CH}_{3}\right), 3.81(\mathrm{~s}$, $\left.3 \mathrm{H}, \mathrm{OCH}_{3}\right), 6.9-7.1(\mathrm{~m}, 4 \mathrm{H}$, olefinic and $\mathrm{ArH}), 7.78-7.9(\mathrm{~m}, 6 \mathrm{H}$, $\left.\mathrm{ArH}, \mathrm{NH}_{2}\right), 8.0(\mathrm{~d}, 2 \mathrm{H}, \mathrm{ArH}, \mathrm{J}=7.0 \mathrm{~Hz})$. ESI-MS: m/z $399(\mathrm{M}+1)$ observed for $\mathrm{C}_{19} \mathrm{H}_{18} \mathrm{~N}_{4} \mathrm{O}_{4} \mathrm{~S}$. 
4-(5-Methyl-4-(3-(thiophen-2-yl)acryloyl)-1H-1,2,3-triazol-1yl)benzene sulfonamide (3d): Yield $98 \%, \mathrm{mp} 350-352{ }^{\circ} \mathrm{C}$. IR spectrum, $v, \mathrm{~cm}^{-1}: 1101,1305,1656,3298,3340 .{ }^{1} \mathrm{H}$ NMR $(400 \mathrm{MHz}$, DMSO- $\left.\mathrm{d}_{6}\right): 2.61\left(\mathrm{~s}, 3 \mathrm{H}, \mathrm{CH}_{3}\right), 7.2(\mathrm{~d}, 1 \mathrm{H}$, olefinic), 7.57-7.65 (m, $4 \mathrm{H}$, $\mathrm{ArH}), 7.71\left(\mathrm{~s}, 2 \mathrm{H}, \mathrm{NH}_{2}\right), 7.8(\mathrm{~d}, 1 \mathrm{H}, \mathrm{ArH}), 7.9(\mathrm{~d}, 2 \mathrm{H}, \mathrm{ArH}), 8.02(\mathrm{~d}, 1 \mathrm{H}$, olefinic). ESI-MS: m/z $375(\mathrm{M}+1)$ observed for $\mathrm{C}_{16} \mathrm{H}_{14} \mathrm{~N}_{4} \mathrm{O}_{3} \mathrm{~S}_{2}$.

\section{4-(4-(3-(Furan-2-yl)acryloyl)-5-methyl-1H-1,2,3-triazol-1-yl)} benzenesulfonamide (3e): Yield 95\%, mp 210-212 ${ }^{\circ} \mathrm{C}$. IR spectrum, $v, \mathrm{~cm}^{-1}: 1163,1344,1654,3255,3334 .{ }^{1} \mathrm{H}$ NMR $(400 \mathrm{MHz}$, DMSO-d 6 ): 2.61 (s, 3H, $\mathrm{CH}_{3}$ ), 7.2 (d, $1 \mathrm{H}$, olefinic), 7.57-7.6 (m, $4 \mathrm{H}$, $\mathrm{ArH}), 7.61\left(\mathrm{~s}, 2 \mathrm{H}, \mathrm{NH}_{2}\right), 7.8$ (d, 1H, ArH), 7.9 (d, 2H, $\left.\mathrm{ArH}\right), 8.02$ (d, 1H, olefinic). ESI-MS: m/z $359(\mathrm{M}+1)$ observed for $\mathrm{C}_{16} \mathrm{H}_{14} \mathrm{~N}_{4} \mathrm{O}_{4} \mathrm{~S}$.

4-(4-(3-(4-(Dimethylamino)phenyl)acryloyl)-5-methyl-1H-1,2,3triazol-1-yl) benzenesulfonamide (3f): Yield $90 \%$, mp $252-254{ }^{\circ} \mathrm{C}$. IR spectrum, $v, \mathrm{~cm}^{-1}: 1134,1357,1653,3253,3334 .{ }^{1} \mathrm{H}$ NMR $(400 \mathrm{MHz}$, DMSO-d 6 ): $2.61\left(\mathrm{~s}, 3 \mathrm{H}, \mathrm{CH}_{3}\right), 2.95\left(\mathrm{~s}, 6 \mathrm{H}, \mathrm{N}\left(\mathrm{CH}_{3}\right)_{2}\right), 7.41-7.44(\mathrm{~m}, 3 \mathrm{H}$, ArH and olefinic), 7.7-7.95 (m, 6H, ArH, $\mathrm{NH}_{2}$, olefinic), 7.96-8.15 (m, $3 \mathrm{H}, \mathrm{ArH})$. ESI-MS: $\mathrm{m} / \mathrm{z} 412(\mathrm{M}+1)$ observed for $\mathrm{C}_{20} \mathrm{H}_{21} \mathrm{~N}_{5} \mathrm{O}_{3} \mathrm{~S}$.

\section{4-(5-Methyl-4-(5-phenyl-4,5-dihydro-1H-pyrazol-3-yl)-1H-} 1,2,3-triazol-1-yl) benzene sulfonamide (4a): Yield 69\%, mp 203$205{ }^{\circ} \mathrm{C}$. IR spectrum, $v, \mathrm{~cm}^{-1}: 761,1165,1346,1592,2370,3259$, 3352. ${ }^{1} \mathrm{H}$ NMR ( $\left.400 \mathrm{MHz}, \mathrm{DMSO}-\mathrm{d}_{6}\right): 2.61\left(\mathrm{~s}, 3 \mathrm{H}, \mathrm{CH}_{3}\right), 3.2(\mathrm{dd}, 1 \mathrm{H}$, $\mathrm{CH}_{2}$ ), 4.1 (dd, $1 \mathrm{H}, \mathrm{CH}_{2}$ ), 5.9 (td, $\left.1 \mathrm{H},-\mathrm{CH}\right), 7.15-7.41$ (m, 5H, $\mathrm{ArH}$ ), 7.6 (s, 2H, NH 2 ), 7.7-7.95 (m, 3H, ArH, NH), 8.0-8.17 (m, 2H, ArH). [13]C NMR (100 MHz, DMSO-d 6 ): $\delta 10.4\left(\mathrm{CH}_{3}\right), 42.3(\mathrm{C}-\mathrm{Py}), 62.2(\mathrm{C}-\mathrm{N})$, 124.5, 125.5, 127.9, 128.4, 128.8, 132.0, 132.3, 139.6, 142.3, 145.8(C$\mathrm{Ar}), \quad 145.2\left(\mathrm{C}_{-} \mathrm{SO}_{2}\right)$. ESI-MS: $\mathrm{m} / \mathrm{z} 383(\mathrm{M}+1)$ observed for $\mathrm{C}_{18} \mathrm{H}_{17} \mathrm{~N}_{6} \mathrm{O}_{2} \mathrm{~S}$, Anal calcd: C, 56.53; H, 4.74; N, 21.97; 0, 8.37; S, 8.38, found: C, 55.53; H, 5.38; N, 20.38, 0, 8.97; S, 9.74;

4-(4-(5-(4-Chlorophenyl)-4,5-dihydro-1H-pyrazol-3-yl)-5-methyl -1H-1,2,3-triazol-1-yl)benzenesulfonamide (4b): Yield 78\%, mp $155-158^{\circ} \mathrm{C}$. IR spectrum, $v, \mathrm{~cm}^{-1}: 836,1161,1306,1593,2372,3259$, 3332. ${ }^{1} \mathrm{H}$ NMR (400 MHz, DMSO-d 6 ); 2.61 (s, 3H,-- $\mathrm{CH}_{3}$ ), 2.9 (dd, $1 \mathrm{H}$,$\left.\mathrm{CH}_{2}\right), 3.6\left(\mathrm{dd}, 1 \mathrm{H},-\mathrm{CH}_{2}\right), 4.85(\mathrm{td}, 1 \mathrm{H},-\mathrm{CH}), 7.4(\mathrm{~m}, 4 \mathrm{H}, \mathrm{ArH}), 7.6(\mathrm{~s}, 2 \mathrm{H},-$ $\mathrm{NH}_{2}$ ), 7.7 (s, 1H,-NH), 7.9 (d, 2H, ArH), 8.1 (d, 2H, ArH). [13]C NMR (100 MHz, DMSO-d 6 ): $\delta 10.4\left(\mathrm{CH}_{3}\right), 42.3$ (C-Py), $62.2(\mathrm{C}-\mathrm{N}), 125.9$, 127.5, 128.8, 128.9, 132.0, 132.3, 138.4, 139.6, 142.3, 143.8 (C-Ar), $145.2\left(\mathrm{C}-\mathrm{SO}_{2}\right)$. ESI-MS: $\mathrm{m} / \mathrm{z} 417(\mathrm{M}+1)$ observed for $\mathrm{C}_{18} \mathrm{H}_{17} \mathrm{ClN}_{6} \mathrm{O}_{2} \mathrm{~S}$, Anal calcd: C, 51.86; H, 4.11; Cl, 8.50; N, 20.16; 0, 7.68; S, 7.69; found: $\mathrm{C}$, $52.11 ; \mathrm{H}, 3.86 ; \mathrm{Cl}, 8.60 ; \mathrm{N}, 21.16 ; 0,7.68 ; \mathrm{S}, 6.59$;

\section{4-(4-(5-(2-Chlorophenyl)-4,5-dihydro-1H-pyrazol-3-yl)-5-} methyl-1H-1,2,3-triazol-1-yl)benzenesulfonamide (4c): Yield 76\%, $\mathrm{mp} 158-160{ }^{\circ} \mathrm{C}$. IR spectrum, $v, \mathrm{~cm}^{-1}: 762,1159,1352,1593,2361$, 3258, 3352. ${ }^{1} \mathrm{H}$ NMR (400 MHz, DMSO-d 6 ): 2.61 (s, 3H,- $\left.\mathrm{CH}_{3}\right), 2.9$ (dd, $\left.1 \mathrm{H},-\mathrm{CH}_{2}\right), 3.6$ (dd, $\left.1 \mathrm{H},-\mathrm{CH}_{2}\right), 4.85$ (td, $\left.1 \mathrm{H},-\mathrm{CH}\right), 7.4(\mathrm{~m}, 4 \mathrm{H}, \mathrm{ArH}), 7.6$ (s, $\left.2 \mathrm{H},-\mathrm{NH}_{2}\right), 7.7(\mathrm{~s}, 1 \mathrm{H},-\mathrm{NH}), 7.9-8.1(\mathrm{~m}, 4 \mathrm{H}, \mathrm{ArH})$. [13] C NMR (100 MHz, DMSO-d 6 ): $\delta 10.4\left(\mathrm{CH}_{3}\right), 42.3$ (C-Py), $62.2(\mathrm{C}-\mathrm{N}), 125.9,127.5,128.8$,

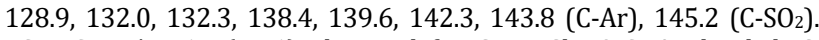
ESI-MS: $\mathrm{m} / \mathrm{z} 417(\mathrm{M}+1)$ observed for $\mathrm{C}_{18} \mathrm{H}_{17} \mathrm{ClN}_{6} \mathrm{O}_{2} \mathrm{~S}$, Anal calcd: C, 51.86; H, 4.11; Cl, 8.50; N, 20.16; 0, 7.68; S, 7.69; found: C, 52.11; H, 3.86; Cl, 8.60; N, 21.16; O, 7.68; S, 6.59;

4-(4-(5-(4-Fluorophenyl)-4,5-dihydro-1H-pyrazol-3-yl)-5-methyl -1H-1,2,3-triazol-1-yl)benzenesulfonamide (4d): Yield 95\%, mp $156-158{ }^{\circ} \mathrm{C}$. IR spectrum, $v, \mathrm{~cm}^{-1}: 839,1167,1305,1594,2361,3265$, 3352. ${ }^{1} \mathrm{H}$ NMR (400 MHz, DMSO-d $) 2.61$ (s, 3H,- $\mathrm{CH}_{3}$ ), 2.9 (dd, $1 \mathrm{H}$,$\mathrm{CH}_{2}$ ), 3.6 (dd, $\left.1 \mathrm{H},-\mathrm{CH}_{2}\right), 4.85$ (td, $\left.1 \mathrm{H},-\mathrm{CH}\right), 7.4(\mathrm{~m}, 4 \mathrm{H}, \mathrm{ArH}), 7.6(\mathrm{~s}, 2 \mathrm{H}$,$\mathrm{NH}_{2}$ ), 7.7 (s, 1H,-NH), 7.9 (d, 2H, ArH), 8.1 (d, 2H, ArH). [13]C NMR (100 MHz, DMSO-d6): $\delta 10.4\left(\mathrm{CH}_{3}\right), 42.3$ (C-Py), 62.2 (C-N), 119.5, 127.5, 128.8, 128.9, 132.0, 132.3, 139.6, 142.3, 143.8 (C-Ar), 145.2 (C$\mathrm{SO}_{2}$ ). 147.5 (C-F). ESI-MS: m/z $401(\mathrm{M}+1)$ observed for $\mathrm{C}_{18} \mathrm{H}_{17} \mathrm{FN}_{6} \mathrm{O}_{2} \mathrm{~S}$, Anal calcd: C, 53.99; H, 4.28; F, 4.74; N, 20.99; 0, 7.99; S, 8.01; found: C, 52.28; H, 5.99; F, 5.00; N, 20.74; 0, 8.99; S, 7.00;

4-(4-(5-(4-Bromophenyl)-4,5-dihydro-1H-pyrazol-3-yl)-5methyl-1H-1,2,3-triazol-1-yl)benzenesulfonamide (4e): Yield $72 \%, \mathrm{mp} 165-167{ }^{\circ} \mathrm{C}$. IR spectrum, $v, \mathrm{~cm}^{-1}: 826,1154,1305,1594$, 2361, 3265, 3352. ${ }^{1} \mathrm{H}$ NMR (400 MHz, DMSO-d 6 ) 2.61 (s, 3H,- $\left.\mathrm{CH}_{3}\right), 2.9$ (dd, $\left.1 \mathrm{H},-\mathrm{CH}_{2}\right), 3.6\left(\mathrm{dd}, 1 \mathrm{H},-\mathrm{CH}_{2}\right), 4.85(\mathrm{td}, 1 \mathrm{H},-\mathrm{CH}), 7.4(\mathrm{~m}, 4 \mathrm{H}, \mathrm{ArH}), 7.6$ $\left(\mathrm{s}, 2 \mathrm{H},-\mathrm{NH}_{2}\right), 7.7(\mathrm{~s}, 1 \mathrm{H},-\mathrm{NH}), 7.9(\mathrm{~d}, 2 \mathrm{H}, \mathrm{ArH}), 8.1(\mathrm{~d}, 2 \mathrm{H}, \mathrm{ArH}) .[13] \mathrm{C}$ NMR (100 MHz, DMSO-d 6 ) $\delta: 10.4\left(\mathrm{CH}_{3}\right), 42.3$ (C-Py), 62.2 (C-N), 124.4, $126.9,127.5,128.8,128.9,132.3,138.4,139.6,142.3,143.8$ (C-Ar),
145.2 (C-SO $)$. ESI-MS: $\mathrm{m} / \mathrm{z} 461(\mathrm{M}+1)$ observed for $\mathrm{C}_{18} \mathrm{H}_{17} \mathrm{BrN}_{6} \mathrm{O}_{2} \mathrm{~S}$, Anal calcd: C, 46.86; H, 3.71; Br, 17.32; N, 18.22; O, 6.94; S, 6.95; found: C, 45.95; H, 4.71; Br, 17.22; N, 18.36; 0, 6.94; S, 6.82;

4-(4-(5-(4-Methoxyphenyl)-4,5-dihydro-1H-pyrazol-3-yl)-5methyl-1H-1,2,3-triazol-1-yl)benzenesulfonamide (4f): Yield 73 $\%$, mp $165-167{ }^{\circ} \mathrm{C}$. IR spectrum, $v, \mathrm{~cm}^{-1}: 1029,1153,1338,1593$, 2362, 3259, 3324. ${ }^{1} \mathrm{H}$ NMR (400 MHz, DMSO-d 6 ) $2.59\left(\mathrm{~s}, 3 \mathrm{H}, \mathrm{CH}_{3}\right), 2.9$ (dd, $1 \mathrm{H}, \mathrm{CH}_{2}$ ), 3.58 (dd, $\left.1 \mathrm{H}, \mathrm{CH}_{2}\right), 3.77\left(\mathrm{~s}, 3 \mathrm{H}, \mathrm{OCH}_{3}\right), 4.8(\mathrm{td}, 1 \mathrm{H}, \mathrm{CH})$, $6.9(\mathrm{~d}, 2 \mathrm{H}, \mathrm{ArH}), 7.3(\mathrm{~d}, 2 \mathrm{H}, \mathrm{ArH}), 7.55-7.65\left(\mathrm{~m}, 3 \mathrm{H}, \mathrm{NH}_{2}, \mathrm{NH}\right), 7.9(\mathrm{~d}$, $2 \mathrm{H}, \mathrm{ArH}), 8.13(\mathrm{~d}, 2 \mathrm{H}, \mathrm{ArH})$. [13]C NMR (100 MHz, DMSO-d6) $\delta: 10.4$ $\left(\mathrm{CH}_{3}\right), 42.3$ (C-Py), $55.6\left(\mathrm{OCH}_{3}\right), 62.2(\mathrm{C}-\mathrm{N}), 123.9,127.5,128.8$, 128.9, 132.0, 132.3, 139.6, 142.3, 143.8 (C-Ar), $145.2\left(\right.$ C-SO $\left._{2}\right), 151.9$ (ArC-OCH $\mathrm{OCH}_{3}$. ESI-MS: m/z $413(\mathrm{M}+1)$ observed for $\mathrm{C}_{19} \mathrm{H}_{20} \mathrm{~N}_{6} \mathrm{O}_{3} \mathrm{~S}$, Anal calcd: C, 55.33; H, 4.89; N, 20.38; 0, 11.64; S, 7.76; found: C, 56.33; H, 3.77; N, 21.64; 0, 10.38; S, 7.88;

4-(5-Methyl-4-(5-(p-tolyl)-4,5-dihydro-1H-pyrazol-3-yl)-1H1,2,3-triazol-1-yl)benzenesulfonamide (4g): Yield $73 \%$, mp 210$213{ }^{\circ} \mathrm{C}$. IR spectrum, $v, \mathrm{~cm}^{-1}: 1153,1352,1596,2362,3259,3324 .{ }^{1} \mathrm{H}$ NMR (400 MHz, DMSO-d 6 ) $2.59\left(\mathrm{~s}, 6 \mathrm{H}, 2-\mathrm{CH}_{3}\right), 2.9\left(\mathrm{dd}, 1 \mathrm{H}, \mathrm{CH}_{2}\right), 3.48$ (dd, $\left.1 \mathrm{H}, \mathrm{CH}_{2}\right), 4.77$ (td, $\left.1 \mathrm{H}, \mathrm{CH}\right), 6.9(\mathrm{~d}, 2 \mathrm{H}, \mathrm{ArH}), 7.3$ (d, $\left.2 \mathrm{H}, \mathrm{ArH}\right)$, 7.55-7.65 (m, 3H, NH $2, \mathrm{NH}), 7.9$ (d, 2H, ArH), 8.13 (d, 2H, ArH). [13]C NMR $\left(100 \mathrm{MHz}\right.$, DMSO-d 6 ) $\delta: 10.4\left(\mathrm{CH}_{3}\right), 21.2,42.3$ (C-Py), 62.2 (CN), 125.9, 127.5, 128.8, 128.9, 132.0, 132.3, 138.4, 139.6, 142.3, 143.8 (C-Ar), $145.2\left(\mathrm{C}-S O_{2}\right)$. ESI-MS: $\mathrm{m} / \mathrm{z} 397(\mathrm{M}+1)$ observed for $\mathrm{C}_{19} \mathrm{H}_{20} \mathrm{~N}_{6} \mathrm{O}_{2} \mathrm{~S}$, Anal calcd: C, 57.56; H, 5.08; N, 21.20; O, 8.07; S, 8.09; found: C, 56.08; H, 6.56; N, 20.09; 0, 9.07; S, 8.20;

4-(5-Methyl-4-(5-(4-nitrophenyl)-4,5-dihydro-1H-pyrazol-3yl)-1H-1,2,3-triazol-1-yl)benzenesulfonamide (4h): Yield $75 \%$, mp 205-207 ${ }^{\circ} \mathrm{C}$. IR spectrum, $v, \mathrm{~cm}^{-1}: 1174,1357,1595,3262,3373$. ${ }^{1} \mathrm{H}$ NMR (400 MHz, DMSO-d $) 2.61\left(\mathrm{~s}, 3 \mathrm{H},-\mathrm{CH}_{3}\right), 2.9$ (dd, $\left.1 \mathrm{H},-\mathrm{CH}_{2}\right), 3.6$ (dd, $\left.1 \mathrm{H},-\mathrm{CH}_{2}\right), 4.85(\mathrm{td}, 1 \mathrm{H},-\mathrm{CH}), 7.4(\mathrm{~m}, 4 \mathrm{H}, \mathrm{ArH}), 7.6\left(\mathrm{~s}, 2 \mathrm{H},-\mathrm{NH}_{2}\right)$, 7.7 (s, 1H,-NH), 7.9 (d, 2H, ArH), 8.1 (d, 2H, ArH). [13]C NMR (100 MHz, DMSO-d 6$)$ 8: $10.4\left(\mathrm{CH}_{3}\right), 42.3$ (C-Py), $62.2(\mathrm{C}-\mathrm{N}), 125.9,127.5$, 128.8, 128.9, 132.0, 132.3, 139.6, 142.3, 143.8(C-Ar), 145.2(C-SO ${ }_{2}$ $149.2\left(\mathrm{C}-\mathrm{NO}_{2}\right)$. ESI-MS: $\mathrm{m} / \mathrm{z} 428(\mathrm{M}+1)$ observed for $\mathrm{C}_{18} \mathrm{H}_{17} \mathrm{~N}_{7} \mathrm{O}_{4} \mathrm{~S}$, Anal calcd: C, 50.58; H, 4.01; N, 22.94; 0, 14.97; S, 7.50; found: C, $50.50 ; \mathrm{H}, 5.58 ; \mathrm{N}, 22.01 ; 0,14.94 ; \mathrm{S}, 6.97$;

4-(4-(5-(2,4-Dimethoxyphenyl)-4,5-dihydro-1H-pyrazol-3-yl)-5methyl-1H-1,2,3-triazol-1-yl)benzenesulfonamide (4i): Yield 65 $\%$, mp $210-212{ }^{\circ} \mathrm{C}$. IR spectrum, $v, \mathrm{~cm}^{-1}: 1174,1357,1594,3232,3363$. ${ }^{1} \mathrm{H}$ NMR (400 MHz, DMSO-d $) 2.61\left(\mathrm{~s}, 3 \mathrm{H},-\mathrm{CH}_{3}\right), 2.9$ (dd, $\left.1 \mathrm{H},-\mathrm{CH}_{2}\right), 3.6$ (dd, $\left.1 \mathrm{H},-\mathrm{CH}_{2}\right), 3.9\left(\mathrm{~s}, 6 \mathrm{H}, 2-\mathrm{OCH}_{3}\right), 4.85(\mathrm{td}, 1 \mathrm{H},-\mathrm{CH}), 7.4(\mathrm{~m}, 4 \mathrm{H}, \mathrm{ArH})$, $7.6\left(\mathrm{~s}, 2 \mathrm{H},-\mathrm{NH}_{2}\right), 7.7(\mathrm{~s}, 1 \mathrm{H},-\mathrm{NH}), 7.9(\mathrm{~d}, 2 \mathrm{H}, \mathrm{ArH}), 8.1(\mathrm{~d}, 2 \mathrm{H}, \mathrm{ArH})$. [13]C NMR (100 MHz, DMSO-d 6 ) $\delta: 10.4\left(\mathrm{CH}_{3}\right), 42.3$ (C-Py), 55.6, 57.4, $62.2(\mathrm{C}-\mathrm{N}), 108.2,110.5,120.2,128.8,128.9,132.0,132.3,139.6,142.3$,

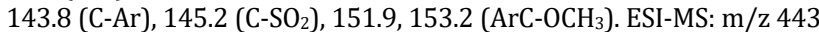
$(\mathrm{M}+1)$ observed for $\mathrm{C}_{20} \mathrm{H}_{22} \mathrm{~N}_{6} \mathrm{O}_{4} \mathrm{~S}$, Anal calcd: C, 54.29; H, 5.01; N, 18.99; 0, 14.46; S, 7.25; found: C, 53.01; H, 6.29; N, 17.46; 0, 14.99; S, 8.25;

4-(4-(5-(4-(Dimethylamino)phenyl)-4,5-dihydro-1H-pyrazol-3yl)-5-methyl-1H-1,2,3-triazol-1-yl)benzenesulfonamide (4j): Yield $62 \%$, mp 220-222 ${ }^{\circ} \mathrm{C}$. IR spectrum, $v, \mathrm{~cm}^{-1}: 1174,1357,1590,3232$, 3373. ${ }^{1} \mathrm{H}$ NMR (400 MHz, DMSO-d 6 ) $2.61\left(\mathrm{~s}, 3 \mathrm{H},-\mathrm{CH}_{3}\right), 2.9$ (s, 6H,$\left.\mathrm{N}\left(\mathrm{CH}_{3}\right)_{2}\right), 3.05\left(\mathrm{dd}, 1 \mathrm{H},-\mathrm{CH}_{2}\right), 3.6\left(\mathrm{dd}, 1 \mathrm{H},-\mathrm{CH}_{2}\right), 4.9(\mathrm{td}, 1 \mathrm{H},-\mathrm{CH}), 7.4(\mathrm{~m}$, $4 \mathrm{H}, \mathrm{ArH}), 7.6\left(\mathrm{~s}, 2 \mathrm{H},-\mathrm{NH}_{2}\right), 7.7(\mathrm{~s}, 1 \mathrm{H},-\mathrm{NH}), 7.9(\mathrm{~d}, 2 \mathrm{H}, \mathrm{ArH}), 8.1(\mathrm{~d}, 2 \mathrm{H}$, $\mathrm{ArH})$. [13]C NMR (100 MHz, DMSO-d 6$) \delta: 10.4\left(\mathrm{CH}_{3}\right), 25.2\left(\mathrm{~N}\left(\mathrm{CH}_{3}\right)_{2}\right)$, 42.3 (C-Py), 62.2 (C-N), 125.9, 127.5, 128.8, 128.9, 132.0, 132.3, 139.6, 141.2, 142.3, 143.8 (C-Ar), 145.2 (C-SO $\left.{ }_{2}\right)$. ESI-MS: m/z $426(\mathrm{M}+1)$ observed for $\mathrm{C}_{20} \mathrm{H}_{23} \mathrm{~N}_{7} \mathrm{O}_{2} \mathrm{~S}$, Anal calcd: C, 56.45; $\mathrm{H}, 5.45 ; \mathrm{N}, 23.04 ; \mathrm{O}$, 7.52; S, 7.54; found: C, 55.45; H, 6.45; N, 22.04; 0, 8.54; S, 7.52;

4-(5-Methyl-4-(5-(thiophen-2-yl)-4,5-dihydro-1H-pyrazol-3-yl)1H-1,2,3-triazol-1-yl)benzenesulfonamide (4k): Yield $95 \%, \mathrm{mp}$ $170-172{ }^{\circ} \mathrm{C}$. IR spectrum, $v, \mathrm{~cm}^{-1}: 1174,1346,1591,3232,3333 .{ }^{1} \mathrm{H}$ NMR (400 MHz, DMSO-d 6 ) 2.58 (s, 3H, CH 3 ), 2.95 (dd, 1H,- $\mathrm{CH}_{2}$ ), 3.6 (dd, $\left.1 \mathrm{H},-\mathrm{CH}_{2}\right), 4.85(\mathrm{td}, 1 \mathrm{H}, \mathrm{CH}), 7.3(\mathrm{~d}, 2 \mathrm{H}, \mathrm{ArH}), 7.42-7.47(\mathrm{~m}, 1 \mathrm{H}$, ArH), 7.6 (s, 2H, NH$)_{2}, 7.7$ (s, $\left.1 \mathrm{H}, \mathrm{NH}\right), 7.9$ (d, 2H, ArH), $8.14(\mathrm{~d}, 2 \mathrm{H}$, $\mathrm{ArH})$. [13]C NMR (100 MHz, DMSO-d 6 ) $\delta: 10.4\left(\mathrm{CH}_{3}\right), 42.3$ (C-Py), 62.2 (C-N), 125.9, 127.5, 128.8, 128.8, 132.0, 132.3, 138.4, 139.6, 142.3, 143.8 (C-Ar), $145.2\left(\mathrm{C}-S O_{2}\right)$. ESI-MS: m/z $389(\mathrm{M}+1)$ observed for $\mathrm{C}_{16} \mathrm{H}_{16} \mathrm{~N}_{6} \mathrm{O}_{2} \mathrm{~S}_{2}$, Anal calcd: C, 49.47; $\mathrm{H}, 4.15 ; \mathrm{N}, 21.63 ; 0,8.24 ; \mathrm{S}$, 16.51; found: C, 48.15; H, 5.47; N, 20.63; O, 8.51; S, 17.24; 
4-(4-(5-(Furan-2-yl)-4,5-dihydro-1H-pyrazol-3-yl)-5-methyl-1H1,2,3-triazol-1-yl)benzenesulfonamide (4l): Yield $78 \%$, mp 190$192{ }^{\circ} \mathrm{C}$. IR spectrum, $v, \mathrm{~cm}^{-1}: 1092,1174,1346,1591,3232,3333 .{ }^{1} \mathrm{H}$ NMR (400 MHz, DMSO-d $\mathrm{d}_{6} 2.58$ (s, 3H, $\left.\mathrm{CH}_{3}\right), 2.95$ (dd, $\left.1 \mathrm{H},-\mathrm{CH}_{2}\right), 3.6$ (dd, $\left.1 \mathrm{H},-\mathrm{CH}_{2}\right), 4.85(\mathrm{td}, 1 \mathrm{H}, \mathrm{CH}), 7.3(\mathrm{~d}, 2 \mathrm{H}, \mathrm{ArH}), 7.42-7.47(\mathrm{~m}, 1 \mathrm{H}$, $\mathrm{ArH}), 7.6$ (s, 2H, NH 2$), 7.7$ (s, 1H, NH), 7.9 (d, 2H, ArH), 8.14 (d, 2H, $\mathrm{ArH})$. [13]C NMR (100 MHz, DMSO-d 6 ) $\delta: 10.4\left(\mathrm{CH}_{3}\right), 42.3$ (C-Py), 62.2 (C-N), 125.95, 127.59, 128.84, 128.84, 132.09, 132.35, 138.45, 139.64, 142.34, 143.81 (C-Ar), 145.28 (C-SO $)$. ESI-MS: $\mathrm{m} / \mathrm{z} 373(\mathrm{M}+1)$ observed for $\mathrm{C}_{16} \mathrm{H}_{16} \mathrm{~N}_{6} \mathrm{O}_{3} \mathrm{~S}$, Anal calcd: C, 51.60; H, 4.33; N, 22.57; O, 12.89; S, 8.61; found: C, 50.33; H, 5.60; N, 21.61; O, 13.89; S, 8.57;

\section{4-(4-(1,5-Diphenyl-4,5-dihydro-1H-pyrazol-3-yl)-5-methyl-1H-} 1,2,3-triazol-1-yl)benzenesulfonamide (5a): Yield $75 \%$, mp 120$122{ }^{\circ} \mathrm{C}$. IR spectrum, $v, \mathrm{~cm}^{-1}: 1163,1336,1593,3192,3223 .{ }^{1} \mathrm{H}$ NMR (400 MHz, DMSO-d $\mathrm{d}_{6}$ ): 2.7 (s, 3H, $\left.\mathrm{CH}_{3}\right), 3.2\left(\mathrm{dd}, 1 \mathrm{H}, \mathrm{CH}_{2}\right), 4.13$ (dd, $1 \mathrm{H}$, $\left.\mathrm{CH}_{2}\right), 5.5(\mathrm{td}, 1 \mathrm{H}, \mathrm{CH}), 6.7(\mathrm{~m}, 1 \mathrm{H}, \mathrm{ArH}), 6.95(\mathrm{~d}, 2 \mathrm{H}, \mathrm{ArH}), 7.15-7.25$ $(\mathrm{m}, 5 \mathrm{H}, \mathrm{ArH}), 7.26-7.7\left(\mathrm{~m}, 8 \mathrm{H}, \mathrm{ArH}, \mathrm{NH}_{2}\right)$. [13]C NMR $(100 \mathrm{MHz}$, DMSO-d 6 ) $\delta: 10.4\left(\mathrm{CH}_{3}\right), 42.3$ (C-Py), $63.2(\mathrm{C}-\mathrm{N}), 123.1,125.1,125.9$, 127.5, 128.8, 128.9, 131.9, 132.0, 132.3, 137.9, 138.4, 139.6, 142.3, 143.8 (Ar-C), $145.2\left(\mathrm{C}^{-S_{2}}\right)_{2}$. ESI-MS: $\mathrm{m} / \mathrm{z} 459(\mathrm{M}+1)$ observed for $\mathrm{C}_{24} \mathrm{H}_{22} \mathrm{~N}_{6} \mathrm{O}_{2} \mathrm{~S}$, Anal calcd: C, 62.86; H, 4.84; N, 18.33; 0, 6.98; S, 6.99; found: $\mathrm{C}, 61.84 ; \mathrm{H}, 5.86 ; \mathrm{N}, 17.98 ; 0,7.99 ; \mathrm{S}, 6.33$;

4-(4-(5-(4-Chlorophenyl)-1-phenyl-4,5-dihydro-1H-pyrazol-3-yl)5-methyl-1H-1,2,3-triazol-1-yl)benzenesulfonamide (5b): Yield 76 $\%, \mathrm{mp} 129-131{ }^{\circ} \mathrm{C}$. IR spectrum, $v, \mathrm{~cm}^{-1}: 756,1163,1338,1596,3213 .{ }^{1} \mathrm{H}$ NMR (400 MHz, DMSO-d $): 2.7$ (s, 3H, $\left.\mathrm{CH}_{3}\right), 3.2$ (dd, 1H, $\left.\mathrm{CH}_{2}\right), 4.13$ (dd, $\left.1 \mathrm{H}, \mathrm{CH}_{2}\right), 5.5$ (td, 1H, CH), $6.7(\mathrm{~m}, 1 \mathrm{H}, \mathrm{ArH}), 6.95(\mathrm{~d}, 2 \mathrm{H}, \mathrm{ArH}), 7.15-7.25$

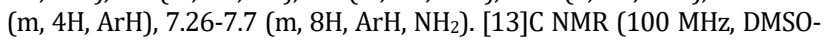
$\left.\mathrm{d}_{6}\right): \delta 10.4\left(\mathrm{CH}_{3}\right), 42.3$ (C-Py), $63.2(\mathrm{C}-\mathrm{N}), 123.1,125.1,125.9,127.5$, $128.9,129.95,131.95,132.09,132.35,137.95,139.5,139.6,142.3,143.8$ (Ar-C), $145.2 \quad\left(\mathrm{C}_{-} \mathrm{SO}_{2}\right)$. ESI-MS: $\mathrm{m} / \mathrm{z} 493 \quad(\mathrm{M}+1)$ observed for $\mathrm{C}_{24} \mathrm{H}_{21} \mathrm{ClN}_{6} \mathrm{O}_{2} \mathrm{~S}$, Anal calcd: C, 58.47; H, 4.29; Cl, 7.19; N, 17.05; 0, 6.50; $\mathrm{S}$, 6.50; found: C, 58.29; H, 4.50; Cl, 7.47; N, 16.05; 0, 7.49; S, 6.20;

4-(4-(5-(4-Fluorophenyl)-1-phenyl-4,5-dihydro-1H-pyrazol-3yl)-5-methyl-1H-1,2,3-triazol-1-yl)benzenesulfonamide (5c): Yield $75 \%, \mathrm{mp} 160-162{ }^{\circ} \mathrm{C}$. IR spectrum, $v, \mathrm{~cm}^{-1}: 832,1163,1332$, 1600, 3233. ${ }^{1} \mathrm{H}$ NMR (400 MHz, DMSO-d $)$ ): 2.7 (s, 3H, $\mathrm{CH}_{3}$ ), 3.2 (dd, $\left.1 \mathrm{H}, \mathrm{CH}_{2}\right), 4.13\left(\mathrm{dd}, 1 \mathrm{H}, \mathrm{CH}_{2}\right), 5.5(\mathrm{td}, 1 \mathrm{H}, \mathrm{CH}), 6.7(\mathrm{~m}, 1 \mathrm{H}, \mathrm{ArH}), 6.95$ (d, $2 \mathrm{H}, \mathrm{ArH}$ ), 7.15-7.25 (m, 4H, $\mathrm{ArH}$ ), 7.26-7.7 (m, 8H, $\mathrm{ArH}, \mathrm{NH}_{2}$ ). [13]C NMR (100 MHz, DMSO-d 6 ): $\delta 10.4\left(\mathrm{CH}_{3}\right), 42.3(\mathrm{C}-\mathrm{Py}), 63.2$ (CN), $119.5,123.1,125.1,127.5,128.8,128.9,131.9,132.0,132.3$, 137.9, 139.6, 142.3, 143.1 (Ar-C), $145.2\left(\mathrm{C}-S O_{2}\right), 147.5$ (C-F). ESI-MS: $\mathrm{m} / \mathrm{z} 477(\mathrm{M}+1)$ observed for $\mathrm{C}_{24} \mathrm{H}_{21} \mathrm{FN}_{6} \mathrm{O}_{2} \mathrm{~S}$, Anal calcd: C, 60.49; $\mathrm{H}$, 4.44; F, 3.99; N, 17.64; 0, 6.72; S, 6.72; found: C, 61.44; H, 3.49; F, $4.64 ; \mathrm{N}, 16.99 ; 0,6.73 ; \mathrm{S}, 6.71$;

4-(4-(5-(4-Bromophenyl)-1-phenyl-4,5-dihydro-1H-pyrazol-3yl)-5-methyl-1H-1,2,3-triazol-1-yl)benzenesulfonamide (5d): Yield $73 \%$ mp $132-134{ }^{\circ} \mathrm{C}$. IR spectrum, $v, \mathrm{~cm}^{-1}: 840,1153,1340$, 1600, 3233. ${ }^{1} \mathrm{H}$ NMR (400 MHz, DMSO-d 6 ): $2.7\left(\mathrm{~s}, 3 \mathrm{H}, \mathrm{CH}_{3}\right), 3.2$ (dd, $\left.1 \mathrm{H}, \mathrm{CH}_{2}\right), 4.13\left(\mathrm{dd}, 1 \mathrm{H}, \mathrm{CH}_{2}\right), 5.5(\mathrm{td}, 1 \mathrm{H}, \mathrm{CH}), 6.7(\mathrm{~m}, 1 \mathrm{H}, \mathrm{ArH}), 6.95$ (d, $2 \mathrm{H}, \mathrm{ArH}), 7.15-7.25(\mathrm{~m}, 4 \mathrm{H}, \mathrm{ArH}), 7.26-7.7\left(\mathrm{~m}, 8 \mathrm{H}, \mathrm{ArH}, \mathrm{NH}_{2}\right) .{ }^{13} \mathrm{C}$ NMR (100 MHz, DMSO-d ${ }_{6}$ ): $\delta$ 10.4 $\left(\mathrm{CH}_{3}\right), 42.3$ (C-Py), $63.2(\mathrm{C}-\mathrm{N})$,
123.1, 124.4, 125.1, 126.9, 127.5, 128.8, 128.9, 131.9, 132.3, 137.9, 138.4, 139.6, 142.3, 143.8 (Ar-C), $145.2\left(\right.$ (C-SO $_{2}$ ). ESI-MS: m/z 537 $(\mathrm{M}+1)$ observed for $\mathrm{C}_{24} \mathrm{H}_{21} \mathrm{BrN}_{6} \mathrm{O}_{2} \mathrm{~S}$, Anal calcd: C, 53.64; $\mathrm{H}, 3.94 ; \mathrm{Br}$, 14.87; N, 15.64; 0, 5.95; S, 5.97; found: C, 53.94; H, 3.97; Br, 15.87; N, $14.64 ; 0,5.94 ; \mathrm{S}, 5.64$;

4-(5-Methyl-4-(5-(4-nitrophenyl)-1-phenyl-4,5-dihydro-1Hpyrazol-3-yl)-1H-1,2,3-triazol-1-yl)benzenesulfonamide (5e): Yield $80 \%$, mp $154-156^{\circ} \mathrm{C}$. IR spectrum, $v, \mathrm{~cm}^{-1}: 1114,1357,1592$, 3192. ${ }^{1} \mathrm{H}$ NMR ( $\left.400 \mathrm{MHz}, \mathrm{DMSO}-\mathrm{d}_{6}\right): 2.59\left(\mathrm{~s}, 3 \mathrm{H}, \mathrm{CH}_{3}\right), 2.9$ (dd, $1 \mathrm{H}$, $\mathrm{CH}_{2}$ ), 3.6 (dd, $1 \mathrm{H}, \mathrm{CH}_{2}$ ), 4.85 (td, $\left.1 \mathrm{H}, \mathrm{CH}\right), 6.95-7.15$ (m, 3H, $\mathrm{ArH}$ ), $7.18(\mathrm{~d}, 2 \mathrm{H}, \mathrm{ArH}), 7.4(\mathrm{~m}, 4 \mathrm{H}, \mathrm{ArH}), 7.6\left(\mathrm{~s}, 2 \mathrm{H}, \mathrm{NH}_{2}\right), 7.7(\mathrm{~d}, 2 \mathrm{H}, \mathrm{ArH})$, 7.9 (d, 2H, ArH). [13]C NMR (100 MHz, DMSO-d 6$): \delta 10.4\left(\mathrm{CH}_{3}\right), 42.3$ (C-Py), 63.2 (C-N), 123.1, 125, 125.9, 127.5, 128.8, 128.9, 131.9,

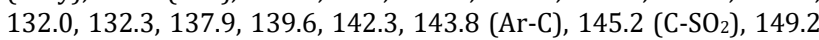
(ArC-NO $)_{2}$. ESI-MS: m/z $504(\mathrm{M}+1)$ observed for $\mathrm{C}_{24} \mathrm{H}_{21} \mathrm{~N}_{7} \mathrm{O}_{4} \mathrm{~S}$, Anal calcd: C, 57.25; H, 4.20; N, 19.47; O, 12.71; S, 6.37; found: C, 56.20; H, $5.25 ; \mathrm{N}, 20.71 ; 0,12.37 ; \mathrm{S}, 5.47$;

4-(4-(5-(4-Methoxyphenyl)-1-phenyl-4,5-dihydro-1H-pyrazol3-yl)-5-methyl-1H-1,2,3-triazol-1-yl)benzenesulfonamide (5f): Yield $78 \%$, mp $125-127^{\circ} \mathrm{C}$. IR spectrum, $v, \mathrm{~cm}^{-1}: 1106,1343,1600$, 3233. ${ }^{1} \mathrm{H}$ NMR ( $\left.400 \mathrm{MHz}, \mathrm{DMSO}-\mathrm{d}_{6}\right): 2.25$ (s, $\left.3 \mathrm{H}, \mathrm{CH}_{3}\right), 3.2$ (dd, $1 \mathrm{H}$, $\mathrm{CH}_{2}$ ), 3.78 (s, $\left.3 \mathrm{H}, \mathrm{OCH}_{3}\right), 4.15$ (dd, $\left.1 \mathrm{H}, \mathrm{CH}_{2}\right), 5.5$ (td, $\left.1 \mathrm{H}, \mathrm{CH}\right), 7.05(\mathrm{~m}$, $1 \mathrm{H}, \mathrm{ArH}), 7.2(\mathrm{~d}, 2 \mathrm{H}, \mathrm{ArH}), 7.3-7.6\left(\mathrm{~m}, 9 \mathrm{H}, \mathrm{ArH}, \mathrm{NH}_{2}\right), 7.85-8.14(\mathrm{~m}$, 3H, ArH). [13]C NMR (100 MHz, DMSO-d 6$): \delta 10.446\left(\mathrm{CH}_{3}\right), 42.3(\mathrm{C}-$ Py), 55.6 $\left(\mathrm{OCH}_{3}\right), 63.2(\mathrm{C}-\mathrm{N}), 123.1,123.9,125.1,127.5,128.8,128.9$, $131.9,132.0,132.3,137.9,139.6,142.3,143.8(\mathrm{Ar}-\mathrm{C}), 145.2\left(\mathrm{C}-\mathrm{SO}_{2}\right)$, $151.9\left(\mathrm{ArC}^{-} \mathrm{OCH}_{3}\right)$. ESI-MS: $\mathrm{m} / \mathrm{z} 489(\mathrm{M}+1)$ observed for $\mathrm{C}_{25} \mathrm{H}_{24} \mathrm{~N}_{6} \mathrm{O}_{3} \mathrm{~S}$, Anal calcd: C, 61.46; H, 4.95; N, 17.20; 0, 9.82; S, 6.57; found: C, 60.56; H, 4.20; N, 17.82; 0, 9.95; S, 7.47;

4-(4-(1-(4-Fluorophenyl)-5-phenyl-4,5-dihydro-1H-pyrazol-3-yl)5-methyl-1H-1,2,3-triazol-1-yl)benzenesulfonamide (5g): Yield 75 $\%$, mp 120-122 ${ }^{\circ} \mathrm{C}$. IR spectrum, $v, \mathrm{~cm}^{-1}: 1174,1357,1689,3192,3273$. ${ }^{1} \mathrm{H}$ NMR (400 MHz, DMSO-d 6 ): 2.65 (s, 3H, $\mathrm{CH}_{3}$ ), 3.15 (dd, $1 \mathrm{H}, \mathrm{CH}_{2}$ ), 4.15 (dd, $\left.1 \mathrm{H}, \mathrm{CH}_{2}\right), 5.38(\mathrm{td}, 1 \mathrm{H}, \mathrm{CH}), 6.9-7.17(\mathrm{~m}, 4 \mathrm{H}, \mathrm{ArH}), 7.5(\mathrm{~d}, 2 \mathrm{H}, \mathrm{ArH})$ 7.6 (s, 2H, NH $\left.\mathrm{N}_{2}\right), 7.8-8.16(\mathrm{~m}, 7 \mathrm{H}, \mathrm{ArH})$. [13]C NMR (100 MHz, DMSO-d 6 ): $\delta 10.4\left(\mathrm{CH}_{3}\right), 42.3$ (C-Py), 63.2 (C-N), 119.5, 123.1, 125.1, 127.5, 128.8, 128.9, 131.9, 132.0, 132.3, 137.9, 139.6, 142.3, 143.8 (Ar-C), 145.2 (C$\mathrm{SO}_{2}$ ), 147.5 (C-F). ESI-MS: $\mathrm{m} / \mathrm{z} 477(\mathrm{M}+1)$ observed for $\mathrm{C}_{24} \mathrm{H}_{21} \mathrm{FN}_{6} \mathrm{O}_{2} \mathrm{~S}$, Anal calcd: C, 60.49; H, 4.44; F, 3.99; N, 17.64; 0, 6.72; S, 6.72; found: C, 61.44; H, 3.49; F, 4.64; N, 16.99; O, 6.73; S, 6.71;

4-(4-(1-(4-Bromophenyl)-5-phenyl-4,5-dihydro-1H-pyrazol-3yl)-5-methyl-1H-1,2,3-triazol-1-yl)benzenesulfonamide (5h): Yield $77 \%$, mp $135-137{ }^{\circ} \mathrm{C}$. IR spectrum, $v, \mathrm{~cm}^{-1}: 856,1163,1338$, 1596, 3213. ${ }^{1} \mathrm{H}$ NMR (400 MHz, DMSO-d 6 ): 2.65 (s, 3H, $\mathrm{CH}_{3}$ ), 3.15 (dd, $\left.1 \mathrm{H}, \mathrm{CH}_{2}\right), 4.15\left(\mathrm{dd}, 1 \mathrm{H}, \mathrm{CH}_{2}\right), 5.38(\mathrm{td}, 1 \mathrm{H}, \mathrm{CH}), 6.9-7.17(\mathrm{~m}, 4 \mathrm{H}$, $\mathrm{ArH}), 7.5(\mathrm{~d}, 2 \mathrm{H}, \mathrm{ArH}), 7.6\left(\mathrm{~s}, 2 \mathrm{H}, \mathrm{NH}_{2}\right), 7.8-8.16(\mathrm{~m}, 7 \mathrm{H}, \mathrm{ArH})$. [13]C NMR $\left(100 \mathrm{MHz}, \mathrm{DMSO}-\mathrm{d}_{6}\right): \delta 10.4\left(\mathrm{CH}_{3}\right), 42.3$ (C-Py), $63.2(\mathrm{C}-\mathrm{N})$, $123.1,124.4,125.1,126.9,127.5,128.8,128.9,131.9,132.3,137.9$, 138.4, 139.6, 142.3, 143.8 (Ar-C), $145.2\left(\mathrm{C}-S O_{2}\right)$. ESI-MS: m/z 537 $(\mathrm{M}+1)$ observed for $\mathrm{C}_{24} \mathrm{H}_{21} \mathrm{BrN}_{6} \mathrm{O}_{2} \mathrm{~S}$, Anal calcd: $\mathrm{C}, 53.64 ; \mathrm{H}, 3.94 ; \mathrm{Br}$, 14.87; N, 15.64; O, 5.95; S, 5.97; found: C, 53.94; H, 3.97; Br, 15.87; N, $14.64 ; 0,5.94 ; \mathrm{S}, 5.64$;

Table 1: Calculated docking scores, binding energies, H-bond count of the targeted compounds inside the PI3K $\alpha$ active site

\begin{tabular}{lll}
\hline Compound & Libdock score & H-Bond count \\
\hline $3 \mathrm{j}$ & 128.405 & 3 \\
$3 \mathrm{i}$ & 145.031 & 4 \\
$3 \mathrm{a}$ & 130.302 & 3 \\
$3 \mathrm{e}$ & 129.849 & 2 \\
$3 \mathrm{~b}$ & 129.803 & 4 \\
$5 \mathrm{~b}$ & 128.22 & 1 \\
$4 \mathrm{~b}$ & 113.056 & 1 \\
$3 \mathrm{~d}$ & 143.264 & 1 \\
$4 \mathrm{~d}$ & 120.09 & 5 \\
$3 \mathrm{l}$ & 125.889 & 3 \\
$4 \mathrm{l}$ & 113.992 & 3 \\
$3 \mathrm{f}$ & 132.497 & 3 \\
$5 \mathrm{f}$ & 130.953 & 1 \\
$4 \mathrm{f}$ & 143.264 & 2 \\
$3 \mathrm{k}$ & 124.359 & 3 \\
$5 \mathrm{i}$ & 132.217 & 0 \\
$4 \mathrm{k}$ & 122.165 & 4 \\
\hline
\end{tabular}


4-(5-Methyl-4-(1-phenyl-5-(thiophen-2-yl)-4,5-dihydro-1Hpyrazol-3-yl)-1H-1,2,3-triazol-1-yl)benzenesulfonamide (5i): Yield $73 \%$, mp 100-102 ${ }^{\circ} \mathrm{C}$. IR spectrum, $v, \mathrm{~cm}^{-1}: 1161,1334,1595$, 3292. ${ }^{1} \mathrm{H}$ NMR ( $\left.400 \mathrm{MHz}, \mathrm{DMSO}-\mathrm{d}_{6}\right): 2.59\left(\mathrm{~s}, 3 \mathrm{H}, \mathrm{CH}_{3}\right), 2.9(\mathrm{dd}, 1 \mathrm{H}$, $\mathrm{CH}_{2}$ ), $3.6\left(\mathrm{dd}, 1 \mathrm{H}, \mathrm{CH}_{2}\right), 4.85$ (td, $\left.1 \mathrm{H}, \mathrm{CH}\right), 6.96-7.14(\mathrm{~m}, 3 \mathrm{H}, \mathrm{ArH})$, $7.18(\mathrm{~d}, 2 \mathrm{H}, \mathrm{ArH}), 7.38(\mathrm{~d}, 2 \mathrm{H}, \mathrm{ArH}), 7.5(\mathrm{~d}, 2 \mathrm{H}, \mathrm{ArH}), 7.55-7.8(\mathrm{~m}, 5 \mathrm{H}$, ArH, $\mathrm{NH}_{2}$ ). [13]C NMR (100 MHz, DMSO-d $): \delta 10.446\left(\mathrm{CH}_{3}\right), 42.36$ (C-Py), 62.29 (C-N), 123.11, 125.11, 125.95, 127.59, 128.84, 128.84, 131.95, 132.09, 132.35, 137.95, 138.45, 139.64, 142.34, 143.81 (ArC), $145.28 \quad\left(\mathrm{C}_{-} \mathrm{SO}_{2}\right)$. ESI-MS: $\mathrm{m} / \mathrm{z} 465 \quad(\mathrm{M}+1)$ observed for $\mathrm{C}_{22} \mathrm{H}_{20} \mathrm{~N}_{6} \mathrm{O}_{2} \mathrm{~S}_{2}$, Anal calcd: C, 56.88; H, 4.34; N, 18.09; 0, 6.89; S, 13.80; found: $\mathrm{C}, 56.34 ; \mathrm{H}, 4.09 ; \mathrm{N}, 18.89 ; \mathrm{O}, 6.80 ; \mathrm{S}, 13.88$;

\section{Results of docking and ADMET}

Table 1 depicts the Libdock scores of top-ranked complex pose per compound along with their binding energies and number of hydrogen bonds. The molecular docking results of synthesized compounds revealed that among all the docked compounds, compound $3 \mathrm{i}$ possessed the high Libdock score of 145.031 compared with the reference ligand KKR exhibiting a Libdock score of 88.35. Docking confirmation of reference ligand KKR with the active site of protein was shown in fig 2 . From the table 1 , it is revealed that all the compounds displayed convincing dock scores ranging from 145.031 to 122.165 , which implies that the designed molecules possess a related mode of binding and valid binding affinity against the protein just as the reference ligand. The compound $3 \mathrm{i}$ is well fitted into the binding site of PI3K $\alpha$ by hydrogen bonds and other close interactions. The interaction of the protein PI $3 \mathrm{~K} \alpha$ and compound $3 \mathrm{i}$ is shown in fig 3 . From results it was observed that four hydrogen bond interactions were presented in the docked pose with compound 3i, two bonds with VAL851 and a single bond with each of the residues GLN859, LYS802. N10 and N11 of the compound $3 \mathrm{i}$ interact with $\mathrm{H}$-atom of the amine group of VAL851 forming two hydrogen bonds (A: VAL851: HN-3i: N10 and A: VAL851: HN-3i: N11) with a hydrogen bond distance of $1.941 \AA$ and $2.359 \AA$ respectively. Third hydrogen bond was formed when the hydrogen atom of GLN859 interacted with the oxygen atom of the compound 3i (A: GLN859:HE22-3i: 023) with a hydrogen bond distance of $1.882 \AA$. Another hydrogen bond was formed between the hydrogen atom of LYS802 and the oxygen atom of the compound 3i (A: LYS802:HZ3-3i: 0) with a hydrogen bond distance of $2.449 \AA$. A few non-bonded interactions were found between the compound $3 \mathrm{i}$ and the residues TYR836, SER854, GLU849, VAL850, ILE932, ILE848, ASP810, ILE800, MET772, ASP933, MET858, and CYS862.

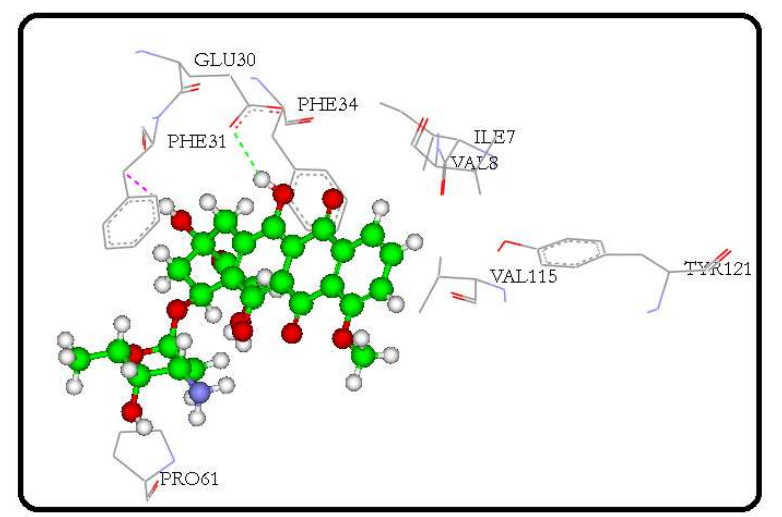

Fig. 2: The docking conformation of reference drug KKR inside the protein PI3K $\alpha$ binding site

The ADMET screening results obtained for synthesized compounds are summarized in table 2 and compared with standard levels. As per discovery studio (DS) parameters, standard comparable values of human intestinal absorption as level 0 , solubility as level 3 and 4 , blood-brain barrier (BBB) penetration as level 3, non-inhibitory property with cytochrome-P(CYP450 2D6) as level 0, and non- toxicity as level 0. An ADMET plot was generated for the blood-brain barrier penetration and the intestinal absorption using descriptors AlogP98 and 2D polar surphase area (PSA) that comprise confidence ellipses of $95 \%$ and $99 \%$. These ellipses elucidate zones where welloccupied compounds are expected to be settled. The compounds are found to be in the range of 95 and $99 \%$ confidence ellipse for both the intestinal absorption and BBB as shown in fig. 4.

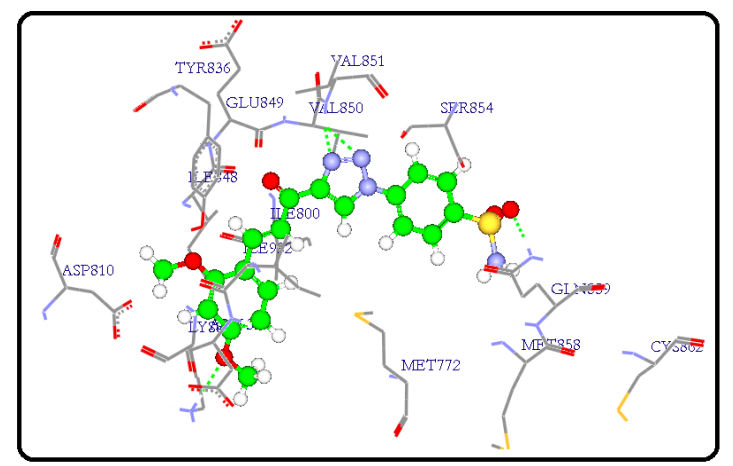

Fig. 3: The hydrogen bond interactions of the compound 3i with the protein PI3K $\alpha$

From the analyzed results, all the synthesized compounds showed a BBB level of 4 except for $4 \mathrm{k}$ which is 3 . The BBB level 4 showing undefined penetration and level 3 indicating a little penetration across the central nervous system (CNS) hence it reduces the side effects linked to CNS. The absorption level was found to be 0 and 1 for all the compounds revealing good and moderate intestinal absorption. For all the compounds, the calculated hepatotoxic level was 1 implying the compounds as toxic. The solubility level 3 indicates very good solubility, level 2 indicates low solubility and level 1 indicates very low solubility or no solubility. All the compounds are found to be having the solubility level 2 except $3 \mathrm{l}$, which was 3 , compound $5 \mathrm{~b}$ was 1 . Similarly, compounds having level 0 were found to be satisfactory with respect to CYP 450 2D6 liver enzyme, suggesting that the compounds are non-inhibitors of the metabolic enzyme and those having level 1 suggests that all the compounds are inhibitors of the metabolic enzyme. Finally, the PPB value found to be 2 for most of the compounds indicates that the compounds have binding $\geq 90 \%$ and the compounds $3 \mathrm{i}, 4 \mathrm{l}$ and $5 \mathrm{k}$ are found to have 0 which denotes that the compounds have binding $\leq 90 \%$ clearly reveal that the compounds have good bioavailability and are not likely to be highly bound to carrier proteins in the blood.



Fig. 4: Plot of PSA versus LogP for candidate compounds showing the 95 and $99 \%$ confidence limit ellipses corresponding to the blood-brain barrier and intestinal absorption models 
Table 2: Predicted ADMET values of synthesised compounds

\begin{tabular}{|c|c|c|c|c|c|c|c|c|}
\hline Name & $\begin{array}{l}\text { BBB } \\
\text { level }\end{array}$ & $\begin{array}{l}\text { Absorption } \\
\text { level }\end{array}$ & $\begin{array}{l}\text { Solubility } \\
\text { level }\end{array}$ & $\begin{array}{l}\text { Hepato } \\
\text { toxicity }\end{array}$ & CYP2D6 & $\begin{array}{l}\text { PPB } \\
\text { level }\end{array}$ & AlogP98 & 2D PSA \\
\hline $3 j$ & 4 & 0 & 2 & 1 & 0 & 2 & 2.757 & 109.665 \\
\hline $3 \mathrm{i}$ & 4 & 1 & 2 & 1 & 0 & 0 & 2.562 & 124.172 \\
\hline $3 a$ & 4 & 0 & 2 & 1 & 0 & 2 & 2.594 & 106.312 \\
\hline $3 e$ & 4 & 0 & 2 & 1 & 0 & 2 & 3.343 & 106.312 \\
\hline $3 b$ & 4 & 0 & 2 & 1 & 0 & 2 & 3.259 & 106.312 \\
\hline $5 b$ & 4 & 1 & 1 & 1 & 1 & 2 & 4.612 & 103.687 \\
\hline $4 b$ & 4 & 0 & 2 & 1 & 0 & 2 & 2.83 & 113.145 \\
\hline $3 d$ & 4 & 1 & 2 & 1 & 0 & 2 & 3.179 & 121.367 \\
\hline $5 c$ & 4 & 0 & 2 & 1 & 0 & 2 & 2.371 & 113.145 \\
\hline 31 & 4 & 0 & 3 & 1 & 0 & 2 & 1.99 & 118.866 \\
\hline 41 & 4 & 0 & 2 & 1 & 0 & 0 & 1.454 & 125.699 \\
\hline $3 f$ & 4 & 0 & 2 & 1 & 0 & 2 & 2.578 & 115.242 \\
\hline $5 f$ & 4 & 1 & 2 & 1 & 1 & 2 & 3.932 & 112.617 \\
\hline $4 \mathrm{f}$ & 4 & 1 & 2 & 1 & 0 & 2 & 3.179 & 121.367 \\
\hline $4 \mathrm{k}$ & 3 & 0 & 2 & 1 & 0 & 2 & 2.32 & 106.312 \\
\hline $5 \mathrm{i}$ & 4 & 0 & 2 & 1 & 1 & 2 & 3.567 & 103.687 \\
\hline $4 \mathrm{k}$ & 4 & 0 & 2 & 1 & 0 & 0 & 1.785 & 113.145 \\
\hline
\end{tabular}

\section{Cytotoxic activity}

Cytotoxic activity results were summarized in table 3 . All tested compounds have shown significant cytotoxic activity.
Among all the tested compounds, $3 \mathrm{~b}$ and $4 \mathrm{~d}$ exhibited significant percentage inhibition in cell proliferation at an $\mathrm{IC}_{50}$ value of $4.54 \mu \mathrm{g} / \mathrm{ml}$ and $7.75 \mu \mathrm{g} / \mathrm{ml}$ as shown in fig. 5 .

Table 3: Cytotoxicity of synthesized compounds on MCF-7 cell line

\begin{tabular}{|c|c|c|}
\hline S. No. & Compound & $\mathrm{IC}_{50}$ value $(\mu \mathrm{g} / \mathrm{ml})$ \\
\hline 1 & $3 b$ & 22.5 \\
\hline 2 & $3 f$ & 15.5 \\
\hline 3 & $3 \mathrm{i}$ & 4.54 \\
\hline 4 & $3 \mathrm{k}$ & 34.3 \\
\hline 5 & 31 & 25.8 \\
\hline 6 & $4 d$ & 7.75 \\
\hline 7 & $4 \mathrm{f}$ & 26 \\
\hline 8 & $4 \mathrm{k}$ & 19.25 \\
\hline 9 & 41 & 55.8 \\
\hline 10 & $5 b$ & 43.4 \\
\hline 11 & Taxol (standard) & $15 \mathrm{nmol}$ \\
\hline
\end{tabular}



Fig. 5: Graphical representation of $\mathrm{IC}_{50}$ values of compounds $3 \mathrm{i}$ and $4 \mathrm{~d}$

Table 4: Effect of compound 4d on total leukocyte count $\left(10^{3} / \mu \mathrm{l}\right)$ in potassium oxonate induced gout in mice

\begin{tabular}{llll}
\hline Groups & On 7th $^{\text {th }}$ & On 14 & On 29y \\
\hline Normal Control & $7.862 \pm 0.729$ & $7.862 \pm 0.729$ & $7.862 \pm 0.729$ \\
Disease Control & $125.0 \pm 5.510$ & $110.0 \pm 3.443$ & $120.9 \pm 7.868$ \\
Standard Control & $101.7 \pm 4.728$ & $77.80 \pm 5.001$ & $24.69 \pm 0.786$ \\
$4 \mathrm{~d}(50 \mathrm{mg} / \mathrm{kg})$ & $129.5 \pm 3.521$ & $97.02 \pm 5.100$ & $62.37 \pm 5.006$ \\
$4 \mathrm{~d}(100 \mathrm{mg} / \mathrm{kg})$ & $107.1 \pm 5.094$ & $66.46 \pm 2.954$ & $13.59 \pm 1.762$ \\
\hline
\end{tabular}

All the data are expressed as mean $\pm \operatorname{SEM}(n=6)$. 
Table 4.1: Effect of compound $4 \mathrm{~d}$ on neutrophils count $\left(10^{3} / \mu \mathrm{l}\right)$ in potassium oxonate induced gout in mice

\begin{tabular}{llll}
\hline Groups & On $7^{\text {th }}$ day & On 14th day $^{\text {th }}$ & 0n 29th day \\
\hline Normal Control & $2.228 \pm 0.3582$ & $2.228 \pm 0.3582$ & $2.228 \pm 0.3582$ \\
Disease Control & $54.98 \pm 2.284$ & $26.73 \pm 2.672$ & $10.50 \pm 0.9429$ \\
Standard Control & $49.12 \pm 3.132$ & $29.48 \pm 2.917$ & $3.237 \pm 0.3677$ \\
$4 \mathrm{~d}(50 \mathrm{mg} / \mathrm{kg})$ & $58.76 \pm 1.151$ & $38.07 \pm 4.850$ & $16.35 \pm 2.999$ \\
$4 \mathrm{~d}(100 \mathrm{mg} / \mathrm{kg})$ & $48.07 \pm 4.850$ & $22.16 \pm 1.875$ & $6.305 \pm 1.037$ \\
\hline
\end{tabular}

All the data are expressed as mean $\pm \operatorname{SEM}(n=6)$.

Table 4.2: Effect of compound 4d on monocyte count $\left(10^{3} / \mu \mathrm{l}\right)$ in potassium oxonate induced gout in mice

\begin{tabular}{llll}
\hline Groups & On $7^{\text {th }}$ day & On 14 $^{\text {th day }}$ & On 29 $^{\text {th }}$ day \\
\hline Normal Control & $0.18 \pm 0.043$ & $0.18 \pm 0.043$ & $0.18 \pm 0.043$ \\
Disease Control & $48.57 \pm 4.362$ & $68.06 \pm 4.847$ & $97.88 \pm 6.664$ \\
Standard Control & $36.72 \pm 2.663$ & $34.19 \pm 2.433$ & $19.07 \pm 1.032$ \\
$4 d(50 \mathrm{mg} / \mathrm{kg})$ & $49.16 \pm 3.324$ & $38.73 \pm 2.883$ & $29.36 \pm 2.665$ \\
$4 \mathrm{~d}(100 \mathrm{mg} / \mathrm{kg})$ & $38.73 \pm 2.883$ & $33.69 \pm 3.270$ & $3.457 \pm 0.418$ \\
\hline
\end{tabular}

All the data are expressed as mean $\pm \operatorname{SEM}(n=6)$.

Table 4.3: Effect of compound 4d on lymphocyte count $\left(10^{3} / \mu \mathrm{l}\right)$ in potassium oxonate induced gout in mice

\begin{tabular}{llll}
\hline Groups & On (th day $^{\text {th }}$ & On 14 $^{\text {th day }}$ & On 29th $^{\text {thay }}$ \\
\hline Normal Control & $5.47 \pm 0.554$ & $5.47 \pm 0.554$ & $5.47 \pm 0.554$ \\
Disease Control & $20.93 \pm 3.005$ & $14.74 \pm 1.792$ & $11.93 \pm 1.313$ \\
Standard Control & $15.64 \pm 1.958$ & $13.91 \pm 1.444$ & $2.338 \pm 0.406$ \\
$4 \mathrm{~d}(50 \mathrm{mg} / \mathrm{kg})$ & $21.06 \pm 1.286$ & $19.98 \pm 2.146$ & $16.35 \pm 3.332$ \\
$4 \mathrm{~d}(100 \mathrm{mg} / \mathrm{kg})$ & $19.98 \pm 2.146$ & $10.38 \pm 0.733$ & $4.910 \pm 0.5303$ \\
\hline
\end{tabular}

All the data are expressed as mean $\pm \operatorname{SEM}(n=6)$.

Table 4.4: Effect of compound 4d on eosinophil count $\left(10^{3} / \mu \mathrm{l}\right)$ in potassium oxonate induced gout in mice

\begin{tabular}{llll}
\hline Groups & On $7^{\text {th }}$ day & On 14 $^{\text {th day }}$ & On 29th $^{\text {tay }}$ \\
\hline Normal Control & $0.03 \pm 0.006$ & $0.03 \pm 0.006$ & $0.03 \pm 0.006$ \\
Disease Control & $0.47 \pm 0.058$ & $0.47 \pm 0.067$ & $0.55 \pm 0.085$ \\
Standard Control & $0.22 \pm 0.034$ & $0.21 \pm 0.036$ & $0.040 \pm 0.013$ \\
4d $(50 \mathrm{mg} / \mathrm{kg})$ & $0.49 \pm 0.043$ & $0.19 \pm 0.040$ & $0.31 \pm 0.085$ \\
4d $100 \mathrm{mg} / \mathrm{kg})$ & $0.29 \pm 0.040$ & $0.21 \pm 0.030$ & $0.24 \pm 0.078$ \\
\hline
\end{tabular}

All the data are expressed as mean $\pm \operatorname{SEM}(n=6)$

Table 4.5: Effect of compound $4 \mathrm{~d}$ on basophil count $\left(10^{3} / \mu \mathrm{l}\right)$ in potassium oxonate induced gout in mice

\begin{tabular}{llll}
\hline Groups & On $7^{\text {th }}$ day & On 14 $^{\text {th }}$ day & On 29th day \\
\hline Normal Control & $0.001 \pm 0.0004$ & $0.001 \pm 0.0004$ & $0.001 \pm 0.0004$ \\
Disease Control & $0.024 \pm 0.006$ & $0.023 \pm 0.008$ & $0.035 \pm 0.008$ \\
Standard Control & $0.029 \pm 0.006$ & $0.023 \pm 0.006$ & $0.003 \pm 0.0009$ \\
$4 d(50 \mathrm{mg} / \mathrm{kg})$ & $0.028 \pm 0.004$ & $0.037 \pm 0.011$ & $0.023 \pm 0.009$ \\
$4 \mathrm{~d}(100 \mathrm{mg} / \mathrm{kg})$ & $0.020 \pm 0.003$ & $0.018 \pm 0.002$ & $0.005 \pm 0.003$ \\
\hline
\end{tabular}

All the data are expressed as mean $\pm \operatorname{SEM}(\mathrm{n}=6)$.

Table 5: Effect of compound 4d on erythrocyte sedimentation rate $(\mathrm{mm} / \mathrm{hr})$ in potassium oxonate induced gout in mice

\begin{tabular}{|c|c|c|c|c|c|}
\hline Animal no & Normal control & Disease control & Standard control & $\begin{array}{l}\text { Compound 4d } \\
(50 \mathrm{mg} / \mathrm{kg})\end{array}$ & $\begin{array}{l}\text { Compound 4d } \\
(100 \mathrm{mg} / \mathrm{kg})\end{array}$ \\
\hline 1 & 1.0 & 8.5 & 2.0 & 3.2 & 2.0 \\
\hline 2 & 2.0 & 5.9 & 4.6 & 4.9 & 4.0 \\
\hline 3 & 0.5 & 9.3 & 2.4 & 6.5 & 1.5 \\
\hline 4 & 2.0 & 10.0 & 3.7 & 7.3 & 2.4 \\
\hline 5 & 1.5 & 8.4 & 4.2 & 6.8 & 1.8 \\
\hline 6 & 1.0 & 11.0 & 1.1 & 5.5 & 0.9 \\
\hline mean \pm SEM & $1.333 \pm 0.2472$ & $8.850 \pm 0.7112$ & $3.000 \pm 0.5615$ & $5.700 \pm 0.6148$ & $2.100 \pm 0.4320$ \\
\hline
\end{tabular}

All the data are expressed as mean $\pm \operatorname{SEM}(n=6)$. 
Table 6: Effect of compound $4 \mathrm{~d}$ on xanthine oxidase $(\mathrm{U} / \mathrm{ml})$ in potassium oxonate induced gout in mice

\begin{tabular}{|c|c|c|c|c|c|}
\hline Animal no & Normal control & Disease control & Standard control & $\begin{array}{l}\text { Compound 4d } \\
(50 \mathrm{mg} / \mathrm{kg})\end{array}$ & $\begin{array}{l}\text { Compound 4d } \\
(100 \mathrm{mg} / \mathrm{kg})\end{array}$ \\
\hline 1 & 1.69 & 4.94 & 1.94 & 1.67 & 1.57 \\
\hline 2 & 0.59 & 2.29 & 1.40 & 3.36 & 1.62 \\
\hline 3 & 1.59 & 3.01 & 2.23 & 2.36 & 1.91 \\
\hline 4 & 1.22 & 2.16 & 0.78 & 2.04 & 1.37 \\
\hline 5 & 1.64 & 2.31 & 1.67 & 3.05 & 1.45 \\
\hline 6 & 0.54 & 4.35 & 1.52 & 4.10 & 2.33 \\
\hline mean \pm SEM & $1.212 \pm 0.2155$ & $3.177 \pm 0.4860$ & $1.590 \pm 0.2029$ & $2.763 \pm 0.3700$ & $1.708 \pm 0.1455$ \\
\hline
\end{tabular}

All the data are expressed as mean $\pm \operatorname{SEM}(n=6)$.

Table 7: Effect of compound 4d on serum creatinine levels $(\mathrm{mg} / \mathrm{dl})$ in potassium oxonate induced gout in mice

\begin{tabular}{|c|c|c|c|c|c|}
\hline Animal no & Normal control & Disease control & Standard control & $\begin{array}{l}\text { Compound 4d } \\
(50 \mathrm{mg} / \mathrm{kg})\end{array}$ & $\begin{array}{l}\text { Compound 4d } \\
\text { (100 mg/kg) }\end{array}$ \\
\hline 1 & 2.5 & 4.69 & 3.21 & 4.03 & 1.44 \\
\hline 2 & 2.0 & 3.38 & 2.94 & 2.89 & 0.96 \\
\hline 3 & 1.5 & 3.23 & 1.06 & 5.23 & 3.50 \\
\hline 4 & 2.5 & 2.61 & 1.72 & 3.12 & 1.70 \\
\hline 5 & 1.0 & 5.30 & 1.39 & 4.27 & 2.40 \\
\hline 6 & 1.0 & 6.01 & 2.58 & 1.98 & 1.30 \\
\hline mean \pm SEM & $1.750 \pm 0.2814$ & $4.203 \pm 0.5437$ & $2.150 \pm 0.3598$ & $3.587 \pm 0.4708$ & $1.883 \pm 0.3787$ \\
\hline
\end{tabular}

All the data are expressed as mean $\pm \operatorname{SEM}(n=6)$.

Table 8: Effect of compound 4d on serum uric acid ( $\mathrm{mg} / \mathrm{dl})$ in potassium oxonate induced gout in mice

\begin{tabular}{|c|c|c|c|c|c|}
\hline Animal no & Normal control & Disease control & Standard control & $\begin{array}{l}\text { Compound 4d } \\
\text { (50 mg/kg) }\end{array}$ & $\begin{array}{l}\text { Compound 4d } \\
\text { (100 mg/kg) }\end{array}$ \\
\hline 1 & 2.74 & 9.65 & 5.50 & 6.69 & 2.68 \\
\hline 2 & 2.82 & 11.17 & 5.57 & 6.38 & 2.94 \\
\hline 3 & 2.1 & 11.01 & 5.37 & 6 & 3.62 \\
\hline 4 & 2.54 & 13.05 & 5.43 & 6.56 & 2.99 \\
\hline 5 & 2.34 & 11.44 & 6.43 & 6.86 & 3.07 \\
\hline 6 & 1.91 & 11.95 & 6.06 & 6.21 & 3.44 \\
\hline mean \pm SEM & $2.408 \pm 0.1466$ & $11.38 \pm 0.4579$ & $5.727 \pm 0.1729$ & $6.450 \pm 0.1295$ & $3.123 \pm 0.1412$ \\
\hline
\end{tabular}

All the data are expressed as mean $\pm \operatorname{SEM}(n=6)$.

Table 9: Effect of compound 4d on blood urea nitrogen $(\mathrm{mg} / \mathrm{dL})$ in potassium oxonate induced gout in mice

\begin{tabular}{|c|c|c|c|c|c|}
\hline Animal no & Normal control & Disease control & Standard control & Compound 4d (50 mg/kg) & Compound 4d (100 mg/kg) \\
\hline 1 & 16.93 & 32.72 & 23.24 & 27.12 & 13.25 \\
\hline 2 & 17.26 & 60.70 & 30.74 & 31.58 & 18.38 \\
\hline 3 & 17.08 & 58.59 & 28.05 & 35.83 & 15.22 \\
\hline 4 & 16.8 & 47.97 & 33.28 & 33.05 & 17.92 \\
\hline 5 & 16.3 & 53.88 & 25.93 & 42.67 & 22.67 \\
\hline 6 & 15.01 & 46.95 & 25.04 & 38.87 & 21.84 \\
\hline mean \pm SEM & $16.56 \pm 0.3379$ & $50.14 \pm 4.144$ & $27.71 \pm 1.534$ & $34.85 \pm 2.251$ & $18.21 \pm 1.491$ \\
\hline
\end{tabular}

All the data are expressed as mean \pm SEM $(n=6)$. Data analysed by one way ANOVA followed by tukeys test. ${ }^{\alpha}<<0.05$, when compared to normal control. $\beta \mathrm{P}<0.05$ when compared to disease control. $\gamma \mathrm{P}<0.05$ when compared to low dose.

\section{CONCLUSION}

In this study, we have designed and synthesized a novel series of pyrazoline incorporated 1, 2, 3-triazole benzene sulphonamides and performed docking simulations in order to identify their binding affinity towards the selected target protein PI3K $\alpha$ and tested for their ADMET profiles. Among all the compounds, compound 3i displayed preferred binding orientations along with strong affinities towards the active site of PI3K $\alpha$ with better ADMET profile. The synthesized compounds were examined for cytotoxic activity and compound 3i exhibited higher activity with an $\mathrm{IC}_{50}$ value of $4.54 \mu \mathrm{g} / \mathrm{ml}$. In Anti-inflammatory activity compound, 4d has significantly shown an anti-inflammatory effect on potassium oxonate induced gout and this was mediated by suppressing the inflammatory responses.

\section{ACKNOWLEDGMENT}

The authors are grateful to G. Pulla Reddy College of Pharmacy and Osmania University and Averin biotech pvt Ltd for their support and providing facilities.

\section{AUTHORS CONTRIBUTIONS}

All authors had equally contributed the work

\section{CONFLICTS OF INTERESTS}

Authors declare no conflicts of interest

\section{REFERENCES}

1. Vander Heiden MG, Cantley LC, Thompson CB. Understanding the warburg effect: the metabolic requirements of cell proliferation. Science 2009;324:1029-33.

2. Fruman DA, Chiu H, Hopkins BD, Bagrodia S, Cantley LC, Abraham RT. The PI3K $\alpha$ pathway in human disease. Cell 2017;170:605-35.

3. De Sousa Mesquita AP, de Araujo Lopes S, Pernambuco Filho PC, Nader HB, Lopes CC. Acquisition of anoikis resistance promotes alterations in the Ras/ERK and PI3K/Akt signaling pathways and matrix remodeling in endothelial cells. Apoptosis 2017;22:1116-37. 
4. Akinleye A, Avvaru P, Furqan $M$, Song $Y$, Liu D. Phosphatidylinositol 3-kinase (PI3K) inhibitors as cancer therapeutics. J Hem Onc 2013;6:88.

5. Cantley LC. The phosphoinositide 3-kinase pathway. Science 2002;296:1655-7.

6. Yamaguchi H, Yoshida S, Muroi E, Yoshida N, Kawamura M, Kouchi Z, et al. Phosphoinositide 3-kinase signaling pathway mediated by $\mathrm{p} 110 \alpha$ regulates invadopodia formation. J Cell Biol 2011;193:1275-88.

7. Rao Jyothi N, Sujith KV, Kalluraya B. An efficient microwaveassisted the synthesis of some novel pyrazoles and their biological activity. Saudi Chem Soc 2008;12:347-52.

8. Rai NS, Kalluraya B, Lingappa B, Shenoy S, Puranic VG. Convenient access to 1, 3, 4-trisubstituted pyrazoles carrying 5-nitrothiophene moiety via 1,3-dipolar cycloaddition of sydnones with acetylenic ketones and their antimicrobial evaluation. Eur J Med Chem 2008;43:1715-20.

9. Sahu SK, Banerjee M, Samantray A, Behera C, Azam MA. Synthesis, analgesic, anti-inflammatory and antimicrobial activities of some novel pyrazoline derivatives. Trop J Pharm Res 2008; 7:961-8.

10. Argade ND, Kalrale BK, Gill CH. Microwave assisted improved method for the synthesis of pyrazole containing 2, 4,disubstituted oxazole-5-one and their antimicrobial activity. J Chem 2008;5:120-9.

11. Chovatia PT, Akabari JD, Kachhadia PK, Zalavadia PD, Joshi HS. Synthesis and selective antitubercular and antimicrobial inhibitory activity of 1-acetyl-3, 5-diphenyl-4, 5-dihydro-(1H)pyrazole derivatives. J Serb Chem Soc 2006;71:713-20.

12. Manna K, Agrawal YK. Microwave assisted synthesis of new indophenazine 1,3,5-trisubstruted pyrazoline derivatives of benzofuran and their antimicrobial activity. Bioorg Med Chem Lett 2009;19:2688-92.

13. Karthikeyan MS, Holla BS, Kumari NS. Synthesis and antimicrobial studies on novel chloro-fluorine containing hydroxy pyrazolines. Eur J Med Chem 2007;42:30-6.

14. Ragavan RV, Vijayakumar V, Kumari NS. Synthesis and antimicrobial activities of novel 1, 5-diaryl pyrazoles. Eur J Med Chem 2010;45:1173-80.

15. Menozzi G, Merello L, Fossa P, Schenone S, Ranise A, Mosti L, et al. Synthesis, antimicrobial activity and molecular modeling studies of halogenated 4-[1H-imidazol-1-yl (phenyl) methyl]-1, 5-diphenyl-1H-pyrazoles. Bioorg Med Chem 2004;12:5465-83.

16. Lee YT, Chung YK. Silver (I)-catalyzed facile synthesis of pyrazoles from propargyl N-sulfonylhydrazones. J Org Chem 2008;73:4698-701.

17. Ezawa M, Garvey DS, Janero DR, Khanapure SP, Letts LG, Martino, et al. Design of a heteroaryl modified 1, 5disubstituted pyrazole cyclooxygenase-2 (COX-2) selective inhibitors. Lett Drug Design Dis 2005;2:40-3.

18. Dmytro Korobko, Dimitra J Hadjipavlou Litina, Liliya Logoyda. Antioxidant and anti-inflammatory properties of a series of new 7,8-disubstituted theophylline containing a pyrazole ring. Asian J Pharm Clin Res 2018;11:448-50.
19. Cocconcelli G, Diodato E, Caricasole A, Gaviraghi G, Genesio E, Ghiron C, et al. Aryl azoles with neuroprotective activity Parallel synthesis and attempts at target identification. Bioorg Med Chem 2008;16:2043-52.

20. Naoum F, Kasiotis KM, Magiatis P, Haroutounian SA. Synthesis of novel nitro-substituted triaryl pyrazole derivatives as potential estrogen receptor ligands. Molecules 2007;12:125973.

21. Manojkumar P, Ravi TK, Gopalakrishnan S. Antioxidant and antibacterial studies of arylazopyrazoles and arylhydrazonopyrazolones containing coumarin moiety. Eur J Med Chem 2009;44:4690-4.

22. Singh MS, Chowdhury S, Koley S. Advances of azide-alkyne cycloaddition-click chemistry over the recent decade. Tetrahedron 2016;72:5257-83.

23. Whiting M, Tripp JC, Lin YC, Lindstrom W, Olson AJ, Elder JH, et al. Rapid discovery and structure-activity profiling of novel inhibitors of human immunodeficiency virus type 1 protease enabled by the copper (I)-catalyzed synthesis of 1, 2, 3triazoles and their further functionalization. J Med Chem 2006;49:7697-710.

24. Kumar BS, Lakshmi PA. Synthesis and molecular docking studies of novel 1, 2, 3-triazole ring-containing 4-(1, 4, 5triphenyl-1H-imidazol-2-yl) phenol derivatives as COX inhibitors. Res Chem Int 2018;44:455-67.

25. Kumar BS, Veena BS, Lakshmi PA, Kamala L, Sujatha E. Synthesis and antimicrobial activity of novel 1, 4, 5-triphenyl1H-imidazol-[1, 2, 3]-triazole derivatives. Russ J Bioorg Chem 2017;43:589-94.

26. Giffin MJ, Heaslet H, Brik A, Lin YC, Cauvi G, Wong CH, et al. A copper (I)-catalyzed 1, 2, 3-triazole azide-alkyne click compound is a potent inhibitor of a multidrug-resistant HIV-1 protease variant. J Med Chem 2008;51:6263-70.

27. Aher NG, Pore VS, Mishra NN, Kumar A, Shukla PK, Sharma A, et al. Synthesis and antifungal activity of 1, 2, 3-triazole containing fluconazole analogues. Bioorg Med Chem Lett 2009;19:759-63.

28. Kumar R, Sharma V, Bua S, Supuran CT, Sharma PK. Synthesis and biological evaluation of benzenesulphonamide-bearing 1,4,5-trisubstituted-1,2,3-triazoles possessing human carbonic anhydrase I, II, IV, and IX inhibitory activity. J Enz Inhi Med Chem 2017;32:1187-94.

29. Prabhu C Jalihala, Vaibhav Rajoriya, Varsha Kashaw. Design, synthesis, and evaluation of a new derivative of 1,2,4-triazoles for antimicrobial and anti-inflammatory activity. Int J Curr Pharm Res 2018;10:29-35.

30. Mousumi Das, Bhaskar Das, Arnab De, Subhasis Banerjee, Amalesh Samanta. Antimicrobial investigation and binding mode analysis of some newly synthesized 4-amino-5-((aryl substituted)-4h-1, 2, 4-triazole-3-yl)-thio linked hydroxamic acid derivatives. Asian J Pharm Clin Res 2019;12:404-6.

31. Mossman T. Rapid colorimetric assay for cellular growth and survival: application to proliferation and cytotoxicity assays. J Immunol Meth 1983;65:55-63. 